\title{
The set of points with Markovian symbolic dynamics for non-uniformly hyperbolic diffeomorphisms
}

\author{
SNIR BEN OVADIA \\ Weizmann Institute of Science, Department of Mathematics, Rehovot, 76100, Israel
}

(Received 22 December 2019 and accepted in revised form 7 August 2020)

\begin{abstract}
The papers [O. M. Sarig. Symbolic dynamics for surface diffeomorphisms with positive entropy. J. Amer. Math. Soc. 26(2) (2013), 341-426] and [S. Ben Ovadia. Symbolic dynamics for non-uniformly hyperbolic diffeomorphisms of compact smooth manifolds. J. Mod. Dyn. 13 (2018), 43-113] constructed symbolic dynamics for the restriction of $C^{r}$ diffeomorphisms to a set $M^{\prime}$ with full measure for all sufficiently hyperbolic ergodic invariant probability measures, but the set $M^{\prime}$ was not identified there. We improve the construction in a way that enables $M^{\prime}$ to be identified explicitly. One application is the coding of infinite conservative measures on the homoclinic classes of Rodriguez-Hertz et al. [Uniqueness of SRB measures for transitive diffeomorphisms on surfaces. Comm. Math. Phys. 306(1) (2011), 35-49].
\end{abstract}

Key words: Markov partition, non-uniform hyperbolicity, symbolic dynamics 2020 Mathematics Subject Classification: 37B10, 37D25 (Primary)

\section{Introduction}

Symbolic dynamics and Markov partitions have played a key role in the theory of uniformly hyperbolic dynamical systems [AW67, Bow08, BR75, PP90, Sin68a, Sin68b, Sin72, Rue04, Rue76].

This paper studies the symbolic dynamics of non-uniformly hyperbolic diffeomorphisms $f: M \rightarrow M$. In this case, the papers [BO18, Sar13] constructed a countable Markov partition for a subset $M^{\prime} \subseteq M$ of the manifold, which carries all 'sufficiently hyperbolic' ergodic invariant probability measures (a precise statement is given in §2). See [Lim20, LM18, LS19] for other coding results in the non-uniformly hyperbolic setup and [Lim19] for a survey of recent advances in the construction of symbolic dynamics for non-uniformly hyperbolic systems.

Although $M^{\prime}$ is 'big' from the point of view of hyperbolic ergodic invariant probability measures, it is usually not equal to the entire manifold. For example, it does not contain elliptic islands, or orbits with zero Lyapunov exponents. 
The papers [BO18, Sar13] do not identify $M^{\prime}$ explicitly. In this paper we modify the construction of the Markov partition done there to yield a countable Markov partition for which the set $M^{\prime}$ can be determined explicitly.

Our main result says that $M^{\prime}$ equals the set of orbits which are summable and recurrently weakly temperable.

Summability and recurrent weak temperability are defined in $\$ 2.2$. The precise statement, Theorem 4.5, is given in $\S 4$. Finally, $\S 5$ adopts the results of [BCS] on transitive codings of homoclinic classes for our setup.

An important motivation for this work is an ongoing project on generalized SRB measures. These are possibly infinite invariant measures with strong 'physical' properties. To study them symbolically, we need to know if they are carried by the set $M^{\prime}$. This does not follow from the results of [BO18, Sar13], which only identify $M^{\prime}$ modulo hyperbolic probability measures.

This paper treats diffeomorphisms. The case of flows brings in new difficulties, because of issues related to singularities in the Poincaré section. The paper [LS19] codes a smaller set than the set of Lyapunov regular points. The author has been informed by Lima that together with Buzzi and Crovisier they now have a coding which captures a larger set than the set of Lyapunov regular orbits [Lim].

\section{Statement of results}

Let $M$ be a compact Riemannian manifold without boundary, of dimension $d \geq 2$. Let $f \in$ $\operatorname{Diff}^{1+\beta}(M), \beta>0$ (i.e. $f$ is invertible, $f, f^{-1}$ are differentiable, and both the derivatives $d_{x} f, d_{x} f^{-1}$ are $\beta$-Hölder continuous functions of $x$ ).

\subsection{General notation.}

(1) For every $a, b \in \mathbb{R}, c \in \mathbb{R}^{+}, a=e^{ \pm c} \cdot b$ means $e^{-c} \cdot b \leq a \leq e^{c} \cdot b$, and $a \wedge b:=$ $\min \{a, b\}$.

(2) For all $x \in M,\langle\cdot, \cdot\rangle_{x}: T_{x} M \times T_{x} M \rightarrow \mathbb{R}$ is the inner product on the tangent space of $x$ given by the Riemannian metric. $|\cdot|_{x}: T_{x} M \rightarrow \mathbb{R}$ is the norm induced by the inner product, $|\xi|_{x}^{2}:=\langle\xi, \xi\rangle_{x}$, for all $\xi \in T_{x} M$. We often omit the $x$ subscript of the inner product and of the norm, when the tangent space in domain is clear from the context.

This work uses tools which were previously developed in [B018, Sar13]. In the following subsection, we introduce two notions of hyperbolic points, in order to have a canonical (in this context, 'canonical' means definitions which do not rely on a specific construction of symbolic dynamics, but which depend only on the quality of hyperbolicity of the orbit of the point) characterization for a set of points which our symbolic extension codes (see [BO18, Sar13]).

2.2. Non-uniform hyperbolicity. Suppose that $\chi>0$.

(1) $\chi$-summability. The set of $\chi$-summable points is

$$
\begin{aligned}
& \chi \text {-summ }:=\left\{x \in M: \text { there exists a splitting } T_{x} M=H^{s}(x) \oplus H^{u}(x)\right. \text { such that } \\
& \left.\sup _{\xi_{s} \in H^{s}(x),\left|\xi_{s}\right|=1} \sum_{m=0}^{\infty}\left|d_{x} f^{m} \xi_{s}\right|^{2} e^{2 \chi m}<\infty, \sup _{\xi_{u} \in H^{u}(x),\left|\xi_{u}\right|=1} \sum_{m=0}^{\infty}\left|d_{x} f^{-m} \xi_{u}\right|^{2} e^{2 \chi m}<\infty\right\} .
\end{aligned}
$$


(2) $\chi$-hyperbolicity. The set of $\chi$-hyperbolic points is

$$
\begin{aligned}
\chi \text {-hyp : }= & \left\{x \in M: \text { there exists a splitting } T_{x} M=H^{s}(x) \oplus H^{u}(x)\right. \text { such that } \\
& \quad \text { for all } \xi_{s} \in H^{s}(x) \backslash\{0\}, \xi_{u} \in H^{u}(x) \backslash\{0\}, \\
& \left.\limsup _{n \rightarrow \infty} \frac{1}{n} \log \left|d_{x} f^{n} \xi_{s}\right|, \limsup _{n \rightarrow \infty} \frac{1}{n} \log \left|d_{x} f^{-n} \xi_{u}\right|<-\chi\right\} .
\end{aligned}
$$

(3) For all $x \in \chi$-hyp, $\chi(x):=-\max \left\{\sup _{\xi_{s} \in H^{s}(x) \backslash\{0\}} \lim \sup _{n \rightarrow \infty}(1 / n) \log \left|d_{x} f^{n} \xi_{s}\right|\right.$, $\left.\sup _{\xi_{u} \in H^{u}(x) \backslash\{0\}} \lim \sup _{n \rightarrow \infty}(1 / n) \log \left|d_{x} f^{-n} \xi_{u}\right|\right\}$.

(4) For all $x \in \chi$-summ, for all $\xi, \eta \in T_{x} M$, write $\xi=\xi_{s}+\xi_{u}, \eta=\eta_{s}+\eta_{u}$ with $\xi_{s}, \eta_{s} \in H^{s}(x), \xi_{u}, \eta_{u} \in H^{u}(x)$,

$$
\begin{aligned}
\left\langle\xi_{s}, \eta_{s}\right\rangle_{x, s}^{\prime} & :=2 \sum_{m=0}^{\infty}\left\langle d_{x} f^{m} \xi_{s}, d_{x} f^{m} \eta_{s}\right\rangle_{x} e^{2 \chi^{m}}, \\
\left\langle\xi_{u}, \eta_{u}\right\rangle_{x, u}^{\prime} & :=2 \sum_{m=0}^{\infty}\left\langle d_{x} f^{-m} \xi_{u}, d_{x} f^{-m} \eta_{u}\right\rangle_{x} e^{2 \chi^{m}} .
\end{aligned}
$$

(5) The Lyapunov metric is $\langle\xi, \eta\rangle_{x}^{\prime}:=\left\langle\xi_{s}, \eta_{s}\right\rangle_{x, s}^{\prime}+\left\langle\xi_{u}, \eta_{u}\right\rangle_{x, u}^{\prime}$.

For each $x \in \chi$-summ, write $s(x):=\operatorname{dim}\left(H^{s}(x)\right), u(x):=\operatorname{dim}\left(H^{u}(x)\right)$. The decomposition $T . M=H^{s}(\cdot) \oplus H^{u}(\cdot)$ may not be unique. However, for $\epsilon$-weakly temperable points and small enough $\epsilon>0$ (see Definition 2.3), the decomposition is unique and invariant. See remark (3) in $\$ 2.4$ for details.

Notice that $\chi$-hyp $\subseteq \chi$-summ. Recall that $x \in M$ is called Lyapunov regular [Pes76], if $T_{x} M=\oplus_{i=1}^{l(x)} H_{i}(x)$, and there exists $\left\{\chi_{i}(x)\right\}_{i=1}^{l(x)}$, such that for all $1 \leq i \leq l(x), \xi \in$ $H_{i}(x) \backslash\{0\}, \lim _{n \rightarrow \pm \infty}(1 / n) \log \left|d_{x} f^{n} \xi\right|=\chi_{i}(x)$, and $\lim _{n \rightarrow \pm \infty}(1 / n) \log \operatorname{Jac}\left(d_{x} f^{n}\right)=$ $\sum_{i=1}^{l(x)} \operatorname{dim}\left(H_{i}(x)\right) \cdot \chi_{i}(x)$. Every Lyapunov regular point $x \in M$ such that $\min _{i}\left\{\left|\chi_{i}(x)\right|\right\}>\chi$ and $\left\{i: \chi_{i}(x)>0\right\},\left\{i: \chi_{i}(x)<0\right\} \neq \varnothing$, is $\chi$-hyperbolic and $\chi$-summable with $H^{s}(x):=\oplus_{i: \chi_{i}(x)<0} H_{i}(x)$ and $H^{u}(x):=\oplus_{i: \chi_{i}(x)>0} H_{i}(x)$. However, the converse is false, since there could be $\chi$-hyperbolic (hence $\chi$-summable) points, for which $\lim _{n \rightarrow+\infty}(1 / n) \log \left|d_{x} f^{n} \xi\right|, \lim _{n \rightarrow-\infty}(1 / n) \log \left|d_{x} f^{n} \xi\right|$ are different (or do not exist) for some $\xi \in H_{i}(x) \backslash\{0\}$, given any decomposition $T_{x} M=\oplus H_{i}(x)$.

THEOREM 2.1. For all $x \in \chi$-summ, there exists $C_{\chi}(x): \mathbb{R}^{d} \rightarrow T_{x} M$ a linear invertible map, such that for all $\xi, \eta \in T_{x} M,\left\langle C_{\chi}^{-1}(x) \xi, C_{\chi}^{-1}(x) \eta\right\rangle_{2}=\langle\xi, \eta\rangle_{x}^{\prime}$, where $\langle\cdot, \cdot\rangle_{2}$ is the Euclidean inner product on $\mathbb{R}^{d}$. In addition,

$$
C_{\chi}^{-1}(f(x)) \circ d_{x} f \circ C_{\chi}(x)=\left(\begin{array}{cc}
D_{s}(x) & 0 \\
0 & D_{u}(x)
\end{array}\right),
$$

where $D_{s}(x), D_{u}(x)$ are square matrices of dimensions $s(x), u(x)$ respectively, and $\left\|D_{s}(x)\right\|,\left\|D_{u}^{-1}(x)\right\| \leq e^{-\chi},\left\|D_{s}^{-1}(x)\right\|,\left\|D_{u}(x)\right\| \leq \kappa$ for some constant $\kappa=\kappa(f, \chi)>1$.

The map $C_{\chi}(x)$ is called the Lyapunov change of coordinates, and this theorem is a version of the Pesin-Oseledec reduction theorem [KM95, Pes76], which we prove in [BO18, Theorem 2.4]. $C_{\chi}(x)$ is unique up to an orthogonal self-mapping of $H^{s}(x)$ 
and of $H^{u}(x)$ for a fixed splitting $H^{s}(x) \oplus H^{u}(x)$. In addition, $x \mapsto C_{\chi}(\cdot)$ can be chosen measurably on $\mathrm{RWT}_{\chi}$ where the splitting $T_{x} M=H^{s}(x) \oplus H^{u}(x)$ is unique (see Definition 2.3 and the remark after Theorem 2.5); details can be found in [BO18, Footnote 1].

$\left\|C_{\chi}^{-1}(x)\right\|$ measures the hyperbolicity of $x$ with respect to the decomposition $T_{x} M=$ $H^{s}(x) \oplus H^{u}(x)$ : the greater it is, the worse the hyperbolicity (slow contraction/expansion on stable/unstable tangent spaces, or small angle between the stable and unstable tangent spaces; see [BO18, Proof of Proposition 4.8, Part 1]).

Definition 2.2. Let $\epsilon>0$ and $x \in \chi$-summ, and define $Q_{\epsilon}(x):=\max \left\{Q \in\left\{e^{-\ell \epsilon / 3}\right\}_{\ell \in \mathbb{N}}\right.$ : $\left.Q \leq\left(1 / 3^{6 / \beta}\right) \epsilon^{90 / \beta}\left\|C_{\chi}^{-1}(x)\right\|^{-48 / \beta}\right\}$.

$Q_{\epsilon}(x)$ is the size of a neighborhood of $x$ where $\psi_{f(x)}^{-1} \circ f \circ \psi_{x} \approx$ linear hyperbolic (see Definition 3.3).

Definition 2.3. A point $x \in \chi$-summ is called $\epsilon$-weakly temperable if there exists $q$ : $\left\{f^{n}(x)\right\}_{n \in \mathbb{Z}} \rightarrow\left\{e^{-\ell \epsilon / 3}\right\}_{l \in \mathbb{N}}$ such that:

(1) $q \circ f / q=e^{ \pm \epsilon}$;

(2) for all $n \in \mathbb{Z}, q\left(f^{n}(x)\right) \leq Q_{\epsilon}\left(f^{n}(x)\right)$.

If, in addition to (1) and (2), $q:\left\{f^{n}(x)\right\}_{n \in \mathbb{Z}} \rightarrow\left\{e^{-\ell \epsilon / 3}\right\}_{\ell \in \mathbb{N}}$ can be chosen to also satisfy

(3) $\lim \sup _{n \rightarrow \pm \infty} q\left(f^{n}(x)\right)>0$,

then we say that $x$ is recurrently $\epsilon$-weakly temperable.

Define $\mathrm{WT}_{\chi}^{\epsilon}:=\{x \in \chi$-summ : $x$ is $\epsilon$-weakly temperable $\}, \quad$ and $\operatorname{RWT}_{\chi}^{\epsilon}:=\{x \in$ $\chi$-summ : $x$ is recurrently $\epsilon$-weakly temperable $\}. \mathrm{WT}_{\chi}^{\epsilon}$ is the set of weakly temperable points, with parameters $\chi, \epsilon>0$, and $\mathrm{RWT}_{\chi}^{\epsilon}$ is the set of recurrently weakly temperable points, with parameters $\chi, \epsilon>0$.

Notice that for all $\chi>0, \epsilon>0, \mathrm{RWT}_{\chi}^{\epsilon} \subseteq \mathrm{WT}_{\chi}^{\epsilon}$.

\subsection{Symbolic dynamics.}

THEOREM 2.4. For all $\chi>0$ such that there exists a periodic hyperbolic point $p \in \chi$-hyp, there exists $\epsilon_{\chi}>0$ (which only depends on $\left.M, f, \beta, \chi\right)$ such that for all $0<\epsilon \leq \epsilon_{\chi}$ there exists a countable and locally finite directed graph $\mathcal{G}=(\mathcal{V}, \mathcal{E})=$ $(\mathcal{V}(\chi, \epsilon), \mathcal{E}(\chi, \epsilon))$ which induces a topological Markov shift $\Sigma=\Sigma(\chi, \epsilon):=\left\{\underline{u} \in \mathcal{V}^{\mathbb{Z}}\right.$ : $\left(u_{i}, u_{i+1}\right) \in \mathcal{E}$, for all $\left.i \in \mathbb{Z}\right\}$ with a map $\pi: \Sigma \rightarrow M$ with the following properties.

(1) $\pi \circ \sigma=f \circ \pi$, where $\sigma: \Sigma \rightarrow \Sigma,(\sigma \underline{u})_{i}:=u_{i+1}, i \in \mathbb{Z}$ (the left-shift).

(2) $\pi$ is a Hölder continuous map with respect to the metric $d(\underline{u}, \underline{v}):=\exp (-\min \{i \geq 0$ : $u_{i} \neq v_{i}$ or $\left.\left.u_{-i} \neq v_{-i}\right\}\right)$.

(3) Let $\Sigma^{\#}:=\left\{\underline{u} \in \Sigma\right.$ : there exists $n_{k}, m_{k} \uparrow \infty$ such that $u_{n_{k}}=u_{n_{0}}, u_{-m_{k}}=u_{-m_{0}}$, for all $k<\geq 0\}$. Then $\pi\left[\Sigma^{\#}\right]$ carries all $f$-invariant, $\chi$-hyperbolic probability measures (i.e. hyperbolic measures with Lyapunov exponents greater than $\chi$ in absolute value). 
This theorem is the content of [Sar13, Theorem 4.16] in dimension 2, and [BO18, Theorem 3.13] in any dimension. $\mathcal{V}$ is a collection of double Pesin charts (see Definition 3.3), which is discrete (every $v \in \mathcal{V}$ is a double Pesin chart of the form $v=\psi_{x}^{p^{s}, p^{u}}$ with $0<p^{s}, p^{u} \leq Q_{\epsilon}(x)$; and discreteness means that for all $\eta>0$ : $\left.\#\left\{v \in \mathcal{V}: v=\psi_{x}^{p^{s}, p^{u}} p^{s} \wedge p^{u}>\eta\right\}<\infty\right)$.

\subsection{Statement of the main result.}

THEOREM 2.5. The construction of $\epsilon_{\chi}, \mathcal{V}(\chi, \epsilon), \Sigma(\chi, \epsilon)$ in Theorem 2.4 can be modified such that, in addition, we get $\pi\left[\Sigma(\chi, \epsilon)^{\#}\right]=\operatorname{RWT}_{\chi}^{\epsilon}$ for all $\left.0<\epsilon \leq \epsilon_{\chi}\right)$.

Remark.

(1) In Definition 2.2, the $48 / \beta$ exponent is an artifact of our proof, and any sufficiently large power of $\left\|C_{\chi}^{-1}(x)\right\|$ in the definition of $Q_{\epsilon}(\cdot)$ suffices. Altering the power changes both the set of coded points, and the coding (see $\S 3.1$ ).

(2) By Pesin's tempered kernel lemma [KM95] (see also [BO18, Claim 2.11]), for all $\epsilon>0$, almost every point is recurrently $\epsilon$-weakly temperable with respect to every invariant probability measure carried by $\chi$-summ.

(3) $Q_{\epsilon}(\cdot)$ depends only on $\epsilon$ and on $\left\|C_{\chi}^{-1}(\cdot)\right\|$, which depends only on the decomposition $T . M=H^{s}(\cdot) \oplus H^{u}(\cdot)$. By equation (1), if $x \in \chi$-summ is also $\epsilon$-weakly temperable (and $\epsilon$ is small with respect to $\chi, \beta$, as imposed by the assumption $\epsilon \leq \epsilon_{\chi}$ from Theorem 2.4), then the decomposition $T_{x} M=H^{s}(x) \oplus H^{u}(x)$ must be unique. Therefore, $Q_{\epsilon}(\cdot)$ is defined canonically for $\epsilon$-weakly temperable points, and does not depend on the choice of $C_{\chi}(\cdot)$. Thus, for all $\epsilon \in\left(0, \epsilon_{\chi}\right], \mathrm{WT}_{\chi}^{\epsilon}$ and $\mathrm{RWT}_{\chi}^{\epsilon}$ are defined canonically.

(4) $\mathrm{RWT}_{\chi}^{\epsilon}$ is of full measure with respect to every invariant probability measure carried by $\chi$-summ.

CLAIM 2.6. For all $\epsilon>0$ and $\epsilon^{\prime} \geq 3 / 2 \epsilon$, $\mathrm{RWT}_{\chi}^{\epsilon} \subseteq \mathrm{RWT}_{\chi}^{\epsilon^{\prime}}$, and $\mathrm{WT}_{\chi}^{\epsilon} \subseteq \mathrm{WT}_{\chi}^{\epsilon^{\prime}}$.

Proof. We prove the statement for $\mathrm{RWT}_{\chi}^{\epsilon}$; the proof for $\mathrm{WT}_{\chi}^{\epsilon}$ is similar. Let $x \in$ $\operatorname{RWT}_{\chi}^{\epsilon}$, and let $q:\left\{f^{n}(x)\right\}_{n \in \mathbb{Z}} \rightarrow(0, \epsilon) \cap\left\{e^{-\epsilon \ell / 3}\right\}_{\ell \in \mathbb{N}}$ be given by the recurrent $\epsilon$-weak temperability of $x$. Define $\widetilde{q}\left(f^{n}(x)\right):=\max \left\{t \in\left\{e^{-\epsilon^{\prime} \ell / 3}\right\}_{\ell \in \mathbb{N}}: t \leq q\left(f^{n}(x)\right)\right\}$. It follows that $\widetilde{q} \circ f / \widetilde{q}=e^{ \pm\left(\epsilon+\epsilon^{\prime} / 3\right)}=e^{ \pm\left(\epsilon^{\prime} / 3+2 \epsilon^{\prime} / 3\right)}=e^{ \pm \epsilon^{\prime}}$. It follows from Definition 2.2 that there exists $\widetilde{b}\left(\epsilon, \epsilon^{\prime}\right)>0$ such that for all $n \in \mathbb{Z}, \widetilde{b}\left(\epsilon, \epsilon^{\prime}\right) \cdot Q_{\epsilon}\left(f^{n}(x)\right) \leq Q_{\epsilon^{\prime}}\left(f^{n}(x)\right)$. Let $b\left(\epsilon, \epsilon^{\prime}\right):=\max \left\{t \in\left\{e^{-\epsilon^{\prime} \ell / 3}\right\}_{\ell \in \mathbb{N}}: t \leq \widetilde{b}\left(\epsilon, \epsilon^{\prime}\right)\right\}$, and define $q^{\prime}\left(f^{n}(x)\right):=b\left(\epsilon, \epsilon^{\prime}\right)$. $\widetilde{q}\left(f^{n}(x)\right), n \in \mathbb{Z}$. In addition, $q^{\prime}\left(f^{n}(x)\right) \geq b\left(\epsilon, \epsilon^{\prime}\right) e^{-\epsilon^{\prime} / 3} \cdot q\left(f^{n}(x)\right)$, for all $n \in \mathbb{Z}$, and thus $\lim \sup _{n \rightarrow \pm \infty} q^{\prime}\left(f^{n}(x)\right)>0$. Since $\left\{e^{-\epsilon^{\prime} \ell / 3}\right\}_{\ell \in \mathbb{N}}$ is closed under multiplication, it follows that $q^{\prime}$ satisfies the assumptions of recurrent $\epsilon^{\prime}$-weak temperability for $x$, and so $x \in \mathrm{RWT}_{\chi}^{\epsilon^{\prime}}$.

\section{Review of [B018, Sar13]}

Throughout this section, $f: M \rightarrow M$ is a $C^{1+\beta}$ diffeomorphism of a compact Riemannian manifold without boundary $M$, and $\chi>0$. 
Definition 3.1. Let $x \in \chi$-summ with $T_{x} M=H^{s}(x) \oplus H^{u}(x)$, and let $C_{\chi}(x)$ be a Lyapunov change of coordinates as in Theorem 2.1. Define, for all $\xi_{s} \in H^{s}(x), \xi_{u} \in$ $H^{u}(x)$,

$$
S^{2}\left(x, \xi_{s}\right):=2 \sum_{m \geq 0}\left|d_{x} f^{m} \xi_{s}\right|^{2} e^{2 \chi m}, \quad U^{2}\left(x, \xi_{u}\right):=2 \sum_{m \geq 0}\left|d_{x} f^{-m} \xi_{u}\right|^{2} e^{2 \chi m}
$$

COROLlaRY 3.2. $\left\|C_{\chi}^{-1}(x)\right\|=\sup _{\xi_{s} \in H^{s}(x), \xi_{u} \in H^{u}(x):\left|\xi_{s}+\xi_{u}\right|=1} \sqrt{S^{2}\left(x, \xi_{s}\right)+U^{2}\left(x, \xi_{u}\right)}$.

See [BO18, Theorem 2.4] for proof.

Since $M$ is compact, there exists $r=r(M)>0$ such that the exponential map $\exp _{x}$ : $\left\{v \in T_{x} M:|v| \leq r\right\} \rightarrow M$ is well defined, smooth, and injective for every $x \in M$. When $\epsilon \leq r$, the following definition is well defined since $C_{\chi}(\cdot)$ is a contraction (see [BO18, Lemma 2.9], [Sar13, Lemma 2.5]).

Definition 3.3. (Pesin charts)

(1) Given $x \in \chi$-summ, the map $\psi_{x}^{\eta}:=\exp _{x} \circ C_{\chi}(x):\left\{v \in T_{x} M:|v| \leq \eta\right\} \rightarrow M, \eta \in$ $\left(0, Q_{\epsilon}(x)\right]$, is called a Pesin chart with center $x$.

(2) A double Pesin chart is an ordered couple $\psi_{x}^{p^{s}, p^{u}}:=\left(\psi_{x}^{p^{s}}, \psi_{x}^{p^{u}}\right)$, where $\psi_{x}^{p^{s}}$ and $\psi_{x}^{p^{u}}$ are Pesin charts with the same map and concentric domains.

(3) Given a collection of Pesin charts $\mathcal{A}$, we say that a double Pesin chart $\psi_{x}^{p^{s}}, p^{u}$ is over $\mathcal{A}$ if $\psi_{x}^{p^{s} \wedge p^{u}} \in \mathcal{A}$ (recall that $\left.a \wedge b:=\min \{a, b\}\right)$.

Since $M$ is compact, there exists $\rho(M)>0$ such that for all $x \in M$, there exist a neighborhood $D \subseteq\left\{x^{\prime} \in M: d\left(x, x^{\prime}\right)<\rho(M)\right\}$ and a smooth map $\Theta_{D}: T D \rightarrow \mathbb{R}^{d}$ such that for all $x \in D,\left.\Theta_{D}\right|_{T_{x} M}$ is a linear isometry. Let $\mathcal{D}$ be a finite cover of $M$ by such neighborhoods (see [Sar13, §3.1]).

Definition 3.4. ( $\epsilon$-overlap condition) Let $\psi_{x}^{p}, \psi_{y}^{q}$ be two Pesin charts. We say that $\psi_{x}^{p}$ $\epsilon$-overlaps $\psi_{y}^{q}$ if

(1) $p / q=e^{ \pm \epsilon}$;

(2) for some $D \in \mathcal{D}, x, y \in D$ and $d(x, y)+\left\|\Theta_{D} \circ C_{\chi}(x)-\Theta_{D} \circ C_{\chi}(y)\right\| \leq p^{4} q^{4}$.

This definition is due to Sarig; see [Sar13, Definition 3.1].

3.1. Main steps of [BO18, Sar13]. There exists $\epsilon_{\chi}>0$ which depends on $M, f, \beta, \chi$, such that for all $\epsilon \in\left(0, \epsilon_{\chi}\right]$ the following statements hold.

(1) A set of hyperbolic points. There exists a set, $N U H_{\chi}^{*}$, of Lyapunov regular $\chi$-hyperbolic points, such that for all $x \in N U H_{\chi}^{*}, \lim _{n \rightarrow \pm \infty}(1 / n) \log \left\|C_{\chi}^{-1}\left(f^{n}(x)\right)\right\|$ $=0$. The definition of $N U H_{\chi}^{*}$ is not important for us, and can be found in [Sar13, BO18, B018, Lemma 2.6, Definition 2.10, Claim 2.11].

(2) Coarse graining. $\mathcal{A}_{\epsilon}$ is a countable collection of Pesin charts with the following properties.t

(a) Discreteness. $\left\{\psi_{x}^{\eta} \in \mathcal{A}_{\epsilon}: \eta>t\right\}$ is finite for all $t>0$. 
(b) Sufficiency. For all $x \in N U H_{\chi}^{*}$, and for every sequence $\left(\eta_{n}\right)_{n \in \mathbb{Z}}$ such that $0<\eta_{n} \leq Q_{\epsilon}\left(f^{n}(x)\right), \eta_{n} \in\left\{e^{-\ell \epsilon / 3}\right\}_{\ell \in \mathbb{N}}$ and $\eta_{n} / \eta_{n+1}=e^{ \pm \epsilon}$ for all $n \in \mathbb{Z}$, there exists a sequence $\left(\psi_{x_{n}}^{\eta_{n}}\right)_{n \in \mathbb{Z}}$ of elements of $\mathcal{A}_{\epsilon}$ such that for all $n \in \mathbb{Z}$ :

(i) $\psi_{x_{n}}^{\eta_{n}} \epsilon$-overlaps $\psi_{f^{n}(x)}^{\eta_{n}}$ and $Q_{\epsilon}\left(f^{n}(x)\right) / Q_{\epsilon}\left(x_{n}\right)=e^{ \pm \epsilon / 3}$;

(ii) $\psi_{x_{n+1}}^{\eta_{n+1}} \epsilon$-overlaps $\psi_{f\left(x_{n}\right)}^{\eta_{n+1}}$;

(iii) $\psi_{x_{n-1}}^{\eta_{n-1}} \epsilon$-overlaps $\psi_{f^{-1}\left(x_{n}\right)}^{\eta_{n-1}}$;

(iv) $\psi_{x_{n}}^{\eta_{n}^{\prime}} \in \mathcal{A}_{\epsilon}$ for all $\eta_{n}^{\prime} \in\left\{e^{-\epsilon \ell / 3}\right\}_{\ell \in \mathbb{N}}$ such that $\eta_{n} \leq \eta_{n}^{\prime} \leq \min \left\{Q_{\epsilon}\left(x_{n}\right), e^{\epsilon} \eta_{n}\right\}$.

See [Sar13, Proposition 3.5] for the existence of $\mathcal{A}_{\epsilon}$.

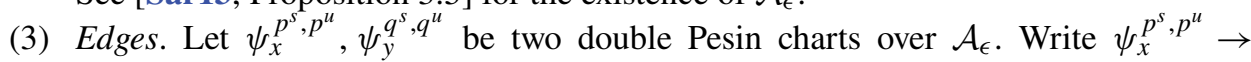
${ }_{\epsilon} \psi_{y}^{q^{s}}, q^{u}$ if:

(a) $\psi_{f(x)}^{q^{s} \wedge q^{u}}$ and $\psi_{y}^{q^{s} \wedge q^{u}} \in$-overlap (recall that $a \wedge b:=\min \{a, b\}$ );

(b) $\psi_{x}^{p^{s} \wedge p^{u}}$ and $\psi_{f^{-1}(y)}^{p^{s} \wedge p^{u}} \epsilon$-overlap;

(c) $q^{u}=\min \left\{e^{\epsilon} p^{u}, Q_{\epsilon}(y)\right\}$ and $p^{s}=\min \left\{e^{\epsilon} q^{s}, Q_{\epsilon}(x)\right\}$;

(d) $\operatorname{dim} H^{s}(x)=\operatorname{dim} H^{s}(y)$ (the decomposition T.M $=H^{s}(\cdot) \oplus H^{u}(\cdot)$ is unique for points in $\left.N U H_{\chi}^{*}\right)$.

This is due to Sarig, [Sar13, Definition 4.1] (see also [BO18, Definition 2.23]).

(4) Vertices and relevance.

(a) A double Pesin chart over $\mathcal{A}_{\epsilon}, \psi_{x}^{p^{s}, p^{u}}$, is $\epsilon$-relevant if there exists a chain of double Pesin charts over $\mathcal{A}_{\epsilon},\left(\psi_{x_{n}}^{p_{n}^{s}, p_{n}^{u}}\right)_{n \in \mathbb{Z}}$, such that $\psi_{x_{0}}^{p_{0}^{s}, p_{0}^{u}}=\psi_{x}^{p^{s}, p^{u}}, p_{n}^{s}, p_{n}^{u} \in$ $\left\{e^{-\epsilon \ell / 3}\right\}_{\ell \in \mathbb{N}}$ and $\psi_{x_{n}}^{p_{n}^{s}, p_{n}^{u}} \rightarrow_{\epsilon} \psi_{x_{n+1}}^{p_{n+1}^{s}, p_{n+1}^{u}}$ for all $n \in \mathbb{Z}$, and there exists $y \in$ $N U H_{\chi}^{*}$ such that $f^{n}(y) \in \psi_{x_{n}}\left[R_{Q_{\epsilon}\left(x_{n}\right)}(0)\right]$ for all $n \in \mathbb{Z}\left(R_{a}(v):=\left\{v^{\prime} \in \mathbb{R}^{d}\right.\right.$ : $\left.\left.\left|v-v^{\prime}\right|_{\infty} \leq a\right\}\right)$.

(b) $\mathcal{V}_{\epsilon}:=\left\{\psi_{x}^{p^{s}, p^{u}}: \psi_{x}^{p^{s} \wedge p^{u}} \in \mathcal{A}_{\epsilon}, p^{s}, p^{u} \in\left\{e^{-\epsilon \ell / 3}\right\}_{\ell \in \mathbb{N}} \cap\left(0, Q_{\epsilon}(x)\right]\right.$, and $\psi_{x}^{p^{s}, p^{u}}$ is $\epsilon$-relevant $\}$ is the set of relevant $\epsilon$-vertices.

(5) Induced graph and TMS. $\mathcal{E}_{\epsilon}:=\left\{\left(v_{1}, v_{2}\right) \in \mathcal{V}_{\epsilon} \times \mathcal{V}_{\epsilon}: v_{1} \rightarrow_{\epsilon} v_{2}\right\} ; \mathcal{G}_{\epsilon}:=\left(\mathcal{V}_{\epsilon}, \mathcal{E}_{\epsilon}\right)$ is a countable directed graph. It is shown in [Sar13, BO18] that $\mathcal{G}_{\epsilon}$ is locally finite: every vertex has finitely many incoming and outgoing edges. We have

$$
\Sigma_{\epsilon}=\Sigma_{\epsilon}\left(\mathcal{G}_{\epsilon}\right):=\left\{\underline{u} \in \mathcal{V}_{\epsilon}^{\mathbb{Z}}:\left(u_{i}, u_{i+1}\right) \in \mathcal{E}_{\epsilon}, \text { for all } i \in \mathbb{Z}\right\} .
$$

(6) This is the induced topological Markov shift (TMS). The local finiteness of $\mathcal{G}_{\epsilon}$ implies that $\Sigma_{\epsilon}$ is locally compact. $\Sigma_{\epsilon}$ admits a factor map $\pi: \Sigma_{\epsilon} \rightarrow M$ as given by Theorem 2.4 .

(7) $\Sigma_{\epsilon}^{\#}:=\left\{\underline{u} \in \Sigma_{\epsilon}\right.$ : there exists $n_{k}, m_{k} \uparrow \infty$ such that $u_{-n_{k}}=u_{-n_{0}}, u_{m_{k}}=u_{m_{0}}$, for all $k \geq 0\}$.

(8) A full measure coded set. $N U H_{\chi}^{\#}:=\left\{x \in N U H_{\chi}^{*}: \lim _{\sup _{n \rightarrow \pm \infty}} q_{\epsilon}\left(f^{n}(x)\right)>0\right\}$, where $q_{\epsilon}(x):=\epsilon / \sum_{n \in \mathbb{Z}} 1 / Q_{\epsilon}\left(f^{n}(x)\right) e^{-|n| / 3 \epsilon}$ is Pesin's tempered kernel [Sar13, B018, §2.5, Definition 2.17]. $N U H_{\chi}^{\#}$ carries every $\chi$-hyperbolic and $f$-invariant probability measure by Poincaré's recurrence theorem. Notice that $N U H_{\chi}^{\#} \subseteq \mathrm{RWT}_{\chi}^{\epsilon}$. By [Sar13, BO18, Theorem 4.16, Theorem 3.13],

$$
\pi\left[\Sigma_{\epsilon}^{\#}\right] \supseteq N U H_{\chi}^{\#} .
$$


(9) Locally finite cover, for all $v \in \mathcal{V}_{\epsilon}, Z(v):=\pi\left[\left\{\underline{u} \in \Sigma_{\epsilon}^{\#}: u_{0}=v\right\}\right] . \mathcal{Z}_{\epsilon}:=\{Z(v)\}_{v \in \mathcal{V}_{\epsilon}}$ has the following properties.

(a) Local finiteness. For all $Z \in \mathcal{Z}_{\epsilon}, \#\left\{Z^{\prime}: Z \cap Z^{\prime} \neq \varnothing\right\}<\infty$ [Sar13, Theorem 10.2].

(b) Covering. $\cup \mathcal{Z}_{\epsilon}=\pi\left[\Sigma_{\epsilon}^{\#}\right] \supseteq N U H_{\chi}^{\#}$.

(10) Countable Markov partition. $\mathcal{R}_{\epsilon}$ is a countable partition given by the Bowen-Sinai refinement of $\mathcal{Z}_{\epsilon}$ (see [Sar13, §11.1]). It has the following properties.

(a) $\mathcal{R}_{\epsilon}$ is a refinement of $\mathcal{Z}_{\epsilon}$ : for all $Z \in \mathcal{Z}_{\epsilon}, R \in \mathcal{R}_{\epsilon}, R \cap Z \neq \varnothing \Rightarrow R \subseteq Z$.

(b) For all $v \in \mathcal{V}_{\epsilon}, \#\left\{R \in \mathcal{R}_{\epsilon}: R_{\epsilon} \subseteq Z(v)\right\}<\infty$ [Sar13, §11].

(c) Symbolic local product structure. For all $R \in \mathcal{R}_{\epsilon}$, for all $x, y \in R$, there exists $z \in R$, such that for all $i \geq 0, R\left(f^{i}(z)\right)=R\left(f^{i}(y)\right), R\left(f^{-i}(z)\right)=R\left(f^{-i}(x)\right)$, where $R(t)$ is the unique partition member of $\mathcal{R}_{\epsilon}$ such that $t \in R(t)$, for $t \in$ $\pi\left[\Sigma_{\epsilon}^{\#}\right]$. We write $z:=[x, y]_{R}$.

(d) $\left\{y \in R:[x, y]_{R}=x\right\} \subseteq W_{\mathrm{loc}}^{s}(x),\left\{y \in R:[y, x]_{R}=x\right\} \subseteq W_{\mathrm{loc}}^{u}(x)$, where $W_{\mathrm{loc}}^{s}(x), W_{\mathrm{loc}}^{u}(x)$ are submanifolds such that for all $z \in W_{\mathrm{loc}}^{s}(x), d\left(f^{k}(z)\right.$, $\left.f^{k}(x)\right) \underset{k \rightarrow \infty}{\stackrel{\text { exponentially fast }}{\longrightarrow}} 0, \quad$ and $\quad$ for $\quad$ all $z \in W_{\mathrm{loc}}^{u}(x), \quad d\left(f^{-k}(z), f^{-k}(x)\right)$ $\underset{k \rightarrow \infty}{\stackrel{\text { exponentially fast }}{\longrightarrow}} 0$.

(e) Symbolic Markov property. Let $R, S \in \mathcal{R}_{\epsilon}$, and let $x \in R \cap f^{-1}[S]$. Let $W^{u}(x, R):=\left\{y \in R: y \in W_{\mathrm{loc}}^{u}(x)\right\}, W^{s}(x, R):=\left\{y \in R: y \in W_{\mathrm{loc}}^{s}(x)\right\}$. Then $f^{-1}\left[W^{u}(f(x), S)\right] \subseteq W^{u}(x, R)$ and $f\left[W^{s}(x, R)\right] \subseteq W^{s}(f(x), S)$.

(11) Induced graph and TMS, with a finite-to-one factor map.

(a) $\widehat{\mathcal{E}}_{\epsilon}:=\left\{(R, S) \in \mathcal{R}_{\epsilon}^{2}\right.$ such that $\left.f^{-1}[S] \cap R \neq \varnothing\right\}, \widehat{\mathcal{G}}_{\epsilon}=\left(\mathcal{R}_{\epsilon}, \widehat{\mathcal{E}}_{\epsilon}\right)$. We have

$$
\widehat{\Sigma}_{\epsilon}=\widehat{\Sigma}_{\epsilon}\left(\widehat{\mathcal{G}_{\epsilon}}\right):=\left\{\underline{R} \in \mathcal{R}_{\epsilon}^{\mathbb{Z}}:\left(R_{i}, R_{i+1}\right) \in \widehat{\mathcal{E}}_{\epsilon} \text {, for all } i \in \mathbb{Z}\right\} .
$$

(b) $\widehat{\pi}: \widehat{\Sigma}_{\epsilon} \rightarrow M, \widehat{\pi}(\underline{R}):=$ unique element of $\bigcap_{n \in \mathbb{Z}} f^{-n}\left[\overline{R_{n}}\right]$ is well defined for all $\underline{R} \in \widehat{\Sigma}_{\epsilon}$ (see [Sar13, BO18, Lemma 12.4, Theorem 1.1]).

(c) $\widehat{\Sigma}_{\epsilon}^{\#}:=\left\{\underline{R} \in \widehat{\Sigma}_{\epsilon}\right.$ : there exists $n_{k}, m_{k} \uparrow \infty$ such that $R_{-n_{k}}=R_{-n_{0}}, R_{m_{k}}=R_{m_{0}}$, for all $k \geq 0\},\left.\widehat{\pi}\right|_{\widehat{\Sigma}_{\epsilon}^{\#}}$ is finite-to-one, and $\widehat{\pi}\left[\widehat{\Sigma}_{\epsilon}^{\#}\right] \supseteq N U H_{\chi}^{\#}$ [Sar13, Theorem 12.5], [BO18, Proof of Theorem 1.1].

3.2. Admissible manifolds. The following two definitions are a modification due to Sarig of a notion introduced by Katok [Kat80], in [Sar13, §4.2, Definition 4.8] (the version here corresponds to the case $d \geq 2$ from [BO18, Definition 3.1, Definition 3.2]). Denote by $R_{a}(b) \subset \mathbb{R}^{c}$ the $|\cdot|_{\infty}$ ball of radius $a$ and center at $b$. Recall the definition of $s(x), u(x)$ from $§ 2.2$.

Definition 3.5. Let $x \in \mathrm{RWT}_{\chi}^{\epsilon}$. A $u$-manifold in $\psi_{x}$ is a manifold $V^{u} \subset M$ of the form

$$
V^{u}=\psi_{x}\left[\left\{\left(F_{1}^{u}\left(t_{s(x)+1}, \ldots, t_{d}\right), \ldots, F_{s(x)}^{u}\left(t_{s(x)+1}, \ldots, t_{d}\right), t_{s(x)+1}, \ldots t_{d}\right):\left|t_{i}\right| \leq q\right\}\right],
$$

where $0<q \leq Q_{\epsilon}(x)$, and $\vec{F}^{u}$ is a $C^{1+\beta / 3}$ function such that $\max \frac{}{R_{q}(0)}\left|\vec{F}^{u}\right|_{\infty} \leq Q_{\epsilon}(x)$.

An $s$-manifold in $\psi_{x}$ is defined similarly as a set

$$
V^{s}=\psi_{x}\left[\left\{\left(t_{1}, \ldots, t_{s(x)}, F_{s(x)+1}^{s}\left(t_{1}, \ldots, t_{s(x)}\right), \ldots, F_{d}^{s}\left(t_{1}, \ldots, t_{s(x)}\right)\right):\left|t_{i}\right| \leq q\right\}\right],
$$


with the same requirements for $\vec{F}^{s}$ and $q$. We will use the superscript $u / s$ in statements which apply to both the $u$ case and the $s$ case. The function $\vec{F}=\vec{F}^{u / s}$ is called the representing function of $V^{u / s}$ at $\psi_{x}$. The parameters of a $u / s$ manifold in $\psi_{x}$ are:

- $\sigma\left(V^{u / s}\right):=\|d \cdot \vec{F}\|_{\beta / 3}:=\max _{\overline{R_{q}(0)}}\|d \cdot \vec{F}\|+\operatorname{Höl}_{\beta / 3}(d \cdot \vec{F}), \quad$ where $\operatorname{Höl}_{\beta / 3}(d . \vec{F}):=$ $\max _{\overrightarrow{t_{1}}, \overrightarrow{t_{2}} \in \overrightarrow{R_{q}(0)}}\left\{\left\|d_{\overrightarrow{t_{1}}} \vec{F}-d_{\overrightarrow{t_{2}}} \vec{F}\right\| /\left|\overrightarrow{t_{1}}-\overrightarrow{t_{2}}\right|^{\beta / 3}\right\}$ and $\|A\|:=\sup _{v \neq 0}|A v|_{\infty} /|v|_{\infty} ;$

- $\quad \gamma\left(V^{u / s}\right):=\left\|d_{0} \vec{F}\right\| ;$

- $\varphi\left(V^{u / s}\right):=|\vec{F}(0)|_{\infty}$;

- $q\left(V^{u / s}\right):=q$.

A $(u / s, \sigma, \gamma, \varphi, q)$-manifold in $\psi_{x}$ is a $u / s$ manifold $V^{u / s}$ in $\psi_{x}$ whose parameters satisfy $\sigma\left(V^{u / s}\right) \leq \sigma, \gamma\left(V^{u / s}\right) \leq \gamma, \varphi\left(V^{u / s}\right) \leq \varphi, q\left(V^{u / s}\right) \leq q$.

Notice that the dimensions of an $s$ - or a $u$-manifold in $\psi_{x}$ depend on $x$. Their sum is $d$.

Definition 3.6. Suppose $x \in \mathrm{RWT}_{\chi}^{\epsilon}$ and $0<p^{s}, p^{u} \leq Q_{\epsilon}(x)$ (i.e. $\psi_{x}^{p^{s}, p^{u}}$ is a double Pesin chart). A $u / s$-admissible manifold in $\psi_{x}^{p^{s}, p^{u}}$ is a $(u / s, \sigma, \gamma, \varphi, q)$-manifold in $\psi_{x}$ such that

$$
\sigma \leq \frac{1}{2}, \quad \gamma \leq \frac{1}{2}\left(p^{u} \wedge p^{s}\right)^{\beta / 3}, \quad \varphi \leq 10^{-3}\left(p^{u} \wedge p^{s}\right), \quad q= \begin{cases}p^{u}, & u \text {-manifolds } \\ p^{s}, & s \text {-manifolds }\end{cases}
$$

CLAIM 3.7. For all $\epsilon>0$ small enough, for all $\underline{u} \in \Sigma_{\epsilon}$, there exist a unique admissible manifold in $u_{0}, V^{u}(\underline{u})$, which depends only on $\left(u_{i}\right)_{i \leq 0}$, and such that $f^{-n}\left[V^{u}(\underline{u})\right] \subseteq$ $u$-admissible manifold in $u_{-n}$ for all $n \geq 0$, and a unique admissible manifold, $V^{s}(\underline{u})$, which depends only on $\left(u_{i}\right)_{i \geq 0}$, and such that $f^{n}\left[V^{s}(\underline{u})\right] \subseteq s$-admissible manifold in $u_{n}$ for all $n \geq 0$. These manifolds satisfy $\{\pi(\underline{u})\}=V^{u}(\underline{u}) \cap V^{s}(\underline{u})$, and this intersection is transversal. $V^{u}(\underline{u})$ and $V^{s}(\underline{u})$ are called the unstable and stable leaves of $\underline{u}$, respectively.

This is the content of [Sar13, Proposition 4.15, Theorem 4.16] and [BO18, Proposition 3.12, Theorem 3.13].

\section{Proof of main result}

We now present the changes to the construction of $\epsilon_{\chi}, \mathcal{V}_{\epsilon}, \Sigma_{\epsilon}$ in [BO18], which will allow us to identify the coded set $\pi\left[\Sigma_{\epsilon}^{\#}\right]$. The main modifications are to replace $N U H_{\chi}^{*}$ by $\mathrm{WT}_{\chi}^{\epsilon}$ in the coarse graining, to replace $N U H^{*}$ by $\operatorname{RWT}_{\chi}^{\epsilon}$ in the definition of relevance, and to replace $\epsilon_{\chi}$ by $\min \left\{\epsilon_{\chi}, \epsilon_{\chi / 2}\right\}$.

Assume that there exists a $\chi$-hyperbolic periodic point $p$, so that there exists $\epsilon_{\chi}>0$ as in Theorem 2.4.

Definition 4.1.

(1) $\stackrel{\star}{\epsilon}_{\chi}:=\min \left\{\epsilon_{\chi / 2}, \epsilon_{\chi}\right\}>0$ (recall that $\epsilon_{\chi}>0$ is introduced in $\S 3.1$ ).

(2) Given $\epsilon \in\left(0, \stackrel{\star}{\epsilon}_{\chi}\right], \stackrel{\star}{\mathcal{A}}_{\epsilon}$ is a discrete and sufficient set of Pesin charts as in $\$ 3.1$ step (2), but given by the coarse graining process for Pesin charts with centers in $\mathrm{WT}_{\chi}^{\epsilon}$, instead of $N U H_{\chi}^{*}$. 
(3) (a) A double Pesin chart over $\stackrel{\star}{\mathcal{A}}_{\epsilon}, \psi_{x}^{p^{s}}, p^{u}$, is called $\star-\epsilon$-relevant if there exists a chain of double Pesin charts over $\stackrel{\mathcal{A}}{\epsilon}_{\epsilon},\left(\psi_{x_{n}}^{p_{n}^{s}, p_{n}^{u}}\right)_{n \in \mathbb{Z}}$, such that $\psi_{x_{0}}^{p_{0}^{s}, p_{0}^{u}}=\psi_{x}^{p^{s}, p^{u}}$, $p_{n}^{s}, p_{n}^{u} \in\left\{e^{-\epsilon \ell / 3}\right\}_{\ell \in \mathbb{N}}$ and $\psi_{x_{n}}^{p_{n}^{s}, p_{n}^{u}} \rightarrow_{\epsilon} \psi_{x_{n+1}}^{p_{n+1}^{s}, p_{n+1}^{u}}$ for all $n \in \mathbb{Z}$, and there exists $y \in \mathrm{RWT}_{\chi}^{\epsilon}$ such that $f^{n}(y) \in \psi_{x_{n}}\left[R_{Q_{\epsilon}\left(x_{n}\right)}(0)\right]$ for all $n \in \mathbb{Z}$ (instead of imposing $y \in N U H_{\chi}^{*}$, as in $\S 3.1$ step (4)(a)).

(b) $\stackrel{\mathcal{V}}{\epsilon}_{\epsilon}:=\left\{\psi_{x}^{p^{s}, p^{u}}: \psi_{x}^{p^{s} \wedge p^{u}} \in \stackrel{\star}{\mathcal{A}}_{\epsilon}, p^{s}, p^{u} \in\left\{e^{-\epsilon \ell / 3}\right\}_{\ell \in \mathbb{N}} \cap\left(0, Q_{\epsilon}(x)\right]\right.$ and $\psi_{x}^{p^{s}}, p^{u}$ is $\star-\epsilon$-relevant $\}$ is the set of $\star-\epsilon$-relevant vertices.

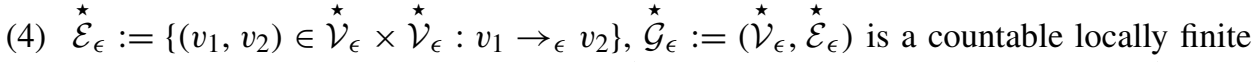
directed graph (the local finiteness of $\stackrel{\star}{\mathcal{G}}_{\epsilon}$ follows from the discreteness of $\mathcal{V}_{\epsilon}$; see $\S 2.3)$.

(5) $\stackrel{\Sigma}{\Sigma}_{\epsilon}:=\left\{\underline{u} \in \hat{\mathcal{V}}_{\epsilon}^{\mathbb{Z}}\right.$ such that $\left(u_{i}, u_{i+1}\right) \in \stackrel{\star}{\mathcal{E}}_{\epsilon}$, for all $\left.i \in \mathbb{Z}\right\}$ is a locally compact TMS induced by $\stackrel{\star}{\mathcal{G}}_{\epsilon}$.

(6) $\stackrel{\star}{\Sigma}_{\epsilon}^{\#}:=\left\{\underline{u} \in \stackrel{\Sigma}{\Sigma}_{\epsilon}\right.$ : there exists $n_{k}, m_{k} \uparrow \infty$ such that $u_{n_{k}}=u_{n_{0}}, u_{-m_{k}}=u_{-m_{0}}$, for all $k \geq 0\}$.

Remark. Notice that the map $\pi: \Sigma_{\epsilon} \rightarrow M$ from Theorem 2.4 is well defined on $\Sigma_{\epsilon}^{\star}$. This is true since, given a chain $\underline{u} \in \Sigma_{\epsilon}, \pi(\underline{u})$ is the unique intersection point of $V^{u}(\underline{u})$ and $V^{s}(\underline{u})$ (see Claim 3.7). The same construction extends to $\Sigma_{\epsilon}^{\star}$, since all arguments which lead to Claim 3.7 for the definition of $V^{s}, V^{u}$ (see [BO18, Theorem 3.6], and the graph transform argument) require only $\chi$-summability of the charts' centers for the Pesin-Oseledec reduction theorem (see Theorem 2.1).

The following lemma is an improvement of [BO18, Lemma 4.5].

LEMMA 4.2. Let $\underline{v} \in \stackrel{\Sigma}{\star}_{\epsilon}$ such that $\pi(\underline{v}) \in \mathrm{RWT}_{\chi}^{\epsilon}$. Write $W^{s}=V^{s}(\underline{v})$ and $z=\pi(\underline{v})$, and let $r \in(\chi / 2, \chi)$. Then there exists $C_{v_{0}, z}>0$ which depends only on $v_{0}, \chi, f, \epsilon$ and $z$ such that for all $y \in W^{s}$,

$$
\sup _{\xi \in T_{y} W^{s},|\xi|=1} \sum_{m \geq 0}\left|d_{y} f^{m} \xi\right|^{2} e^{2 r m} \leq C_{v_{0}, z} \cdot e^{4 \sqrt{\epsilon}}\left\|C_{\chi}^{-1}(z)\right\|^{2} .
$$

Notice that the bound on the right-hand side is uniform in $y$ and $r$, which is the main improvement compared to [BO18, Lemma 4.5].

Proof. Let $q_{\epsilon}:\left\{f^{n}(z)\right\}_{n \in \mathbb{Z}} \rightarrow(0, \epsilon)$ be given by the $\epsilon$-weak temperability of $z$. By definition, $q_{\epsilon}\left(f^{n}(z)\right) \leq Q_{\epsilon}\left(f^{n}(z)\right)$, for all $n \in \mathbb{Z}$. So, by [Sar13, Lemma 4.6], there exists $\left(p_{n}^{s}\right)_{n \in \mathbb{Z}},\left(p_{n}^{u}\right)_{n \in \mathbb{Z}} \subseteq\left\{e^{-\epsilon \ell / 3}\right\}_{\ell \geq 0}$ such that $q_{\epsilon}\left(f^{n}(z)\right) \leq p_{n}^{s} \wedge p_{n}^{u} \leq Q_{\epsilon}\left(f^{n}(z)\right)$, for all $n \in \mathbb{Z}$, and $\underline{u}:=\left(\psi_{f^{i}(z)}^{p_{s}^{s}, p_{i}^{u}}\right)_{i \in \mathbb{Z}}$ is a chain (i.e. $u_{i} \rightarrow_{\epsilon} u_{i+1}$, for all $i \in \mathbb{Z}$, recall $\S 3.1$ step (3)). Notice that the sequence $\left(p_{n}^{s} \wedge p_{n}^{u}\right)_{n \in \mathbb{Z}}$ is $\epsilon$-weakly temperable and recurrent (see [Sar13, Lemma 4.4]). Let $y \in W^{s}$.

The idea of the proof follows the steps of [BO18, Lemma 4.5] for push-forwards of $y, z$ (with $r$ replacing $\chi$ and $\chi$ replacing $\chi_{z}$, and $\epsilon$-weak temperability replacing the assumption 
$\left.\lim _{n \rightarrow \infty}(1 / n) \log \left\|C_{\chi}^{-1}\left(f^{n}(z)\right)\right\|=0\right)$; and then uses a version of [BO18, Lemma 4.6] to pull back the refined bounds. The reason why this works for $r \in(\chi / 2, \chi)$, and not just for $\chi$ as required in [BO18, Lemma 4.6], is that there is no distortion of bounds by the non-complete overlap of charts in the chain $\underline{u}$, as $\underline{u}$ is a chain over a real orbit and not merely a pseudo-orbit.

Set $N=N\left(v_{0}, z\right) \in \mathbb{N}$ such that $N:=\min \left\{N^{\prime} \in \mathbb{N}_{0}: f^{N^{\prime}}\left[W^{s}\right] \subseteq V^{s}\left(\sigma^{N^{\prime}} \underline{u}\right)\right\}$ (which exists since $\operatorname{diam}_{f^{n}\left[W^{s}\right]}\left(f^{n}\left[W^{s}\right]\right)$ decreases exponentially faster than $\left\{q_{\epsilon}\left(f^{n}(z)\right)\right\}_{n \geq 0}$, by $\epsilon$-weak temperability of $z$ ).

Part 1. First we assume $N=0$. Let $c>0$ and $n_{k} \uparrow \infty$ such that $q_{\epsilon}\left(f^{n_{k}}(z)\right) \geq c$ for all $k \geq 0$.

As in the proof of [BO18, Lemma 4.5], let

$$
\begin{aligned}
& n_{z, r}:=\min \left\{n \geq 0: \text { for all } m \geq n, \text { for all } y^{\prime} \in f^{m}\left[W^{s}\right], \text { for all } \xi^{\prime} \in T_{y^{\prime}} f^{m}\left[W^{s}\right],\right. \\
& \left.\qquad\left|\xi^{\prime}\right|=1, \text { for all } j \geq 0,\left|d_{y^{\prime}} f^{j} \xi^{\prime}\right| \leq 2 e^{-j(2 r+\chi) / 3}\left\|C_{\chi}^{-1}\left(f^{m}(z)\right)\right\|\right\} .
\end{aligned}
$$

The reason why the minimum is not over an empty set is that $\operatorname{diam}_{f^{n}\left[W^{s}\right]}\left(f^{n}\left[W^{s}\right]\right)$ decreases exponentially faster than $q_{\epsilon}\left(f^{n}(z)\right.$ ) (by $\epsilon$-weak temperability), and that $z$ is $\chi$-summable, and so $r^{\prime}$-hyperbolic for all $r^{\prime} \in(r, \chi)$. For full details, see the claim within the proof of [BO18, Lemma 4.5], with $r$ replacing $\chi$ and $\chi$ replacing $\chi_{z}$; the assumption $\lim _{n \rightarrow \infty}(1 / n) \log \left\|C_{\chi}^{-1}\left(f^{n}(z)\right)\right\|=0$ is replaced by the $\epsilon$-weak temperability of $z$.

Let $k_{z, r} \geq 0$ such that for all $k \geq k_{z, r}, n_{k} \geq n_{z, r}$. Thus, for all $k \geq k_{z, r}$, for all $y^{\prime} \in$ $f^{n_{k}}\left[W^{s}\right]$

$$
\sup _{\xi^{\prime} \in T_{y^{\prime}} f^{n}\left[W^{s}\right],\left|\xi^{\prime}\right|=1} \sum_{m \geq 0}\left|d_{y^{\prime}} f^{m} \xi^{\prime}\right|^{2} e^{2 r m} \leq \frac{4}{c^{2}} \sum_{m \geq 0} e^{-m(2(\chi-r)) / 3}<\infty .
$$

The bound on the right-hand side depends on $r$ and $z$, but does not depend on $k$ (as long as $k \geq k_{z, r}$ ).

We now wish to use [BO18, Lemma 4.6] with the chain $\underline{u}$, and gain a bound for points in $W^{s}$, instead of points in $f^{n_{k}}\left[W^{s}\right]$. This requires justification, since in [BO18, Lemma 4.6] the exponential factor in the sum is $\chi$, and we wish to apply it to a sum with an exponential factor of $r$. The proof of [BO18, Lemma 4.6] starts by breaking the quotient which we wish to estimate into two factors (see equation (14) in the proof). The first factor is the improvement in the quotient which wish to bound, due to the pull-back by $f^{-1}$. The estimates of the first factor (see equation (17) in the proof) remain unchanged (since $\left.\left\|C_{r}^{-1}\left(f^{n_{k}}(z)\right)\right\| \leq\left\|C_{\chi}^{-1}\left(f^{n_{k}}(z)\right)\right\|\right)$. The second factor is due to the distortion by non-complete overlap of charts (which does not apply to our case since $\underline{u}$ is a chain over the actual orbit of $z$, and not merely a pseudo-orbit, hence the overlap is complete), and due to the distance in the tangent bundle between the tangent vectors $\pi_{z} \xi$ and $\eta$ (in the notation of the proof of [BO18, Lemma 4.6]; see steps 1 and 2 in the proof). The assumption $N=0$ (i.e. $W^{S} \subseteq V^{s}(\underline{u})$ ) allows the estimates of the distance in the tangent bundle to remain as in the proof.

Thus, we may apply [BO18, Lemma 4.6] to the chain $\underline{u}$. Since the bound in equation (2) is uniform in $k$, we may apply [BO18, Lemma 4.6] as many times as we need, starting from $u_{n_{k}}$ where $k$ is as large as we wish. Each time we apply [BO18, Lemma 4.6], the quotient 
which we wish to estimate either improves, or is bounded by $e^{ \pm \sqrt{\epsilon}}$, and every time we hit an element of $\left\{u_{n_{j}}\right\}$ it improves by a definite amount. Since we are free to choose $k$ as large as we wish, eventually it will drop below $e^{4 \sqrt{\epsilon}}$ and will stay there. It follows that

$$
\begin{aligned}
\sup _{\xi \in T_{y} W^{s},|\xi|=1} \sum_{m \geq 0}\left|d_{y} f^{m} \xi\right|^{2} e^{2 r m} & \leq e^{4 \sqrt{\epsilon}} \sup _{\eta \in T_{z} W^{s},|\eta|=1} \sum_{m \geq 0}\left|d_{z} f^{m} \eta\right|^{2} e^{2 r m} \\
& \leq e^{4 \sqrt{\epsilon}}\left\|C_{r}^{-1}(z)\right\|^{2} \leq e^{4 \sqrt{\epsilon}}\left\|C_{\chi}^{-1}(z)\right\|^{2} .
\end{aligned}
$$

Part 2. We now wish to treat the case where $N>0$. Notice that, for all $y \in W^{s}, \xi \in$ $T_{y} W^{s}$,

$$
\begin{aligned}
\sum_{m \geq 0}\left|d_{y} f^{m} \eta\right|^{2} e^{2 r m} & =\sum_{m=0}^{N-1}\left|d_{y} f^{m} \xi\right|^{2} e^{2 r m}+e^{2 r N}\left|d_{y} f^{N} \xi\right|^{2} \sum_{m \geq 0}\left|d_{f^{N}(y)} f^{m} \frac{d_{y} f^{N} \xi}{\left|d_{y} f^{N} \xi\right|}\right|^{2} e^{2 r m} \\
& \leq 2 N\left(M_{f} e^{\chi}\right)^{2 N} \cdot \sum_{m \geq 0}\left|d_{f^{N}(y)} f^{m} \frac{d_{y} f^{N} \xi}{\left|d_{y} f^{N} \xi\right|}\right|^{2} e^{2 r m} .
\end{aligned}
$$

Set $C_{v_{0}, z}:=2 N\left(M_{f} e^{\chi}\right)^{2 N}$, where $N=N\left(v_{0}, z\right)$. Thus, together with part 1 , we get

$$
\sup _{y \in W^{s}} \sup _{\xi \in T_{y} W^{s},|\xi|=1} \sum_{m \geq 0}\left|d_{y} f^{m} \xi\right|^{2} e^{2 r m} \leq C_{v_{0}, z} \cdot e^{4 \sqrt{\epsilon}}\left\|C_{\chi}^{-1}(z)\right\|^{2} .
$$

COROLLARY 4.3. Under the assumptions of Lemma 4.2, for all $y \in W^{s}$,

$$
\sup _{\xi \in T_{y} W^{s},|\xi|=1} \sum_{m \geq 0}\left|d_{y} f^{m} \xi\right|^{2} e^{2 \chi m} \leq C_{v_{0}, z} \cdot e^{4 \sqrt{\epsilon}}\left\|C_{\chi}^{-1}(z)\right\|^{2} .
$$

Proof. Assume by contradiction that there exist $\delta>0, \quad y \in W^{s}, \quad \xi \in T_{y} W^{s}$ such that $\sum_{m=0}^{\infty}\left|d_{y} f^{m} \xi\right|^{2} e^{2 \chi m} \geq C_{v_{0}, z} \cdot e^{4 \sqrt{\epsilon}+\delta}\left\|C_{\chi}^{-1}(z)\right\|^{2}$. Then choose $N>0$ such that $\sum_{m=0}^{N}\left|d_{y} f^{m} \xi\right|^{2} e^{2 \chi m} \geq C_{v_{0}, z} \cdot e^{4 \sqrt{\epsilon}+3 / 4 \delta}\left\|C_{\chi}^{-1}(z)\right\|^{2}$. Choose $\chi / 2 \leq r<\chi$ such that $\sum_{m=0}^{N}\left|d_{y} f^{m} \xi\right|^{2} e^{2 r m} \geq C_{v_{0}, z} \cdot e^{4 \sqrt{\epsilon}+1 / 2 \delta}\left\|C_{\chi}^{-1}(z)\right\|^{2}$, whence $\sum_{m=0}^{\infty}\left|d_{y} f^{m} \xi\right|^{2} e^{2 r m} \geq$ $C_{v_{0}, z} \cdot e^{4 \sqrt{\epsilon}+1 / 2 \delta}\left\|C_{\chi}^{-1}(z)\right\|^{2}$. This is a contradiction to Lemma 4.2.

LEMMA 4.4. Let $\underline{u} \in \stackrel{\Sigma}{\star}_{\epsilon}^{\#}$. Then $x:=\pi(\underline{u})$ is $\chi$-summable, and

$$
\sup _{\xi_{s} \in T_{x} V^{s}(\underline{u}),\left|\xi_{s}\right|=1} \sum_{m \geq 0}\left|d_{x} f^{m} \xi_{s}\right|^{2} e^{2 \chi m} \leq e^{4 \epsilon^{1 / 2}}\left\|C_{\chi}^{-1}\left(x_{0}\right)\right\|^{2}
$$

where $x_{0}$ is the center of $u_{0}=\psi_{x_{0}}^{p_{0}^{s}, p_{0}^{u}}$. A similar claim holds for $V^{u}(\underline{u})$.

Proof. This lemma is the same as Claim 2 in the proof of [BO18, Lemma 4.7], except for the fact that in [BO18, Lemma 4.7] we have $\Sigma^{\#}$ instead of $\Sigma_{\epsilon}^{\star}$. We will now explain how to modify the argument of [BO18, Lemma 4.7] to handle $\Sigma_{\epsilon}^{\star}$. The point is to deal with $\star-\epsilon$-relevance (see Definition 4.1), instead of $\epsilon$-relevance (see $\$ 3.1$ ). 
Assume without loss of generality that there exists $n_{k} \uparrow \infty$ such that $u_{n_{k}}=u_{0}$ for all $k \geq 0$. By the $\star-\epsilon$-relevance of $u_{0}$, there exists a chain $\underline{w} \in \Sigma^{\star} \Sigma_{\epsilon} \cap\left[u_{0}\right]$ such that $z:=\pi(\underline{w}) \in \mathrm{RWT}_{\chi}^{\epsilon}$. This way, we may use Corollary 4.3 to replace [BO18, Lemma 4.5] in the proof of [BO18, Lemma 4.7] (i.e. to bound $S(\cdot, \cdot)$ uniformly on the unit tangent bundle of $\left.V^{s}(\underline{w})\right)$. The statement of Claim 2 in the proof of [BO18, Lemma 4.7] gives what we wanted to show.

THEOREM 4.5. For all $\epsilon \in\left(0, \stackrel{\star}{\epsilon}_{\chi}\right], \pi\left[\stackrel{\star}{\Sigma}_{\epsilon}^{\#}\right]=\mathrm{RWT}_{\chi}^{\epsilon}$.

Proof. Let $\epsilon \in\left(0, \stackrel{\star}{\epsilon}_{\chi}\right]$. The following steps are done both in [BO18], and in [Sar13] when $d=2$.

Step 1. We claim that $\pi\left[\stackrel{\Sigma}{\Sigma}_{\epsilon}^{\#}\right] \supseteq \mathrm{RWT}_{\chi}^{\epsilon}$. The proof is the same as the proof of [B018, Proposition 2.30], except that we have to use $q_{\epsilon}(x)$ given by the definition of recurrent $\epsilon$-weak temperability instead of Pesin's tempered kernel $q_{\epsilon}(x)=$ $\epsilon / \sum_{m \in \mathbb{Z}} 1 / Q_{\epsilon}\left(f^{m}(x)\right) e^{-|m| \epsilon / 3}$.

Step 2. Let $\underline{u} \in \stackrel{\Sigma}{\star}_{\epsilon}^{\#}$, and write $z:=\pi(\underline{u})$. By Lemma 4.4, $z \in \chi$-summ. It is left to show that $z$ is recurrently $\epsilon$-weakly temperable.

Step 3. In order to show recurrent $\epsilon$-weak temperability, we wish to compare $\left\|C_{\chi}^{-1}\left(f^{n}(z)\right)\right\|$ with $\left\|C_{\chi}^{-1}\left(x_{n}\right)\right\|, n \in \mathbb{Z}$, where $u_{n}=\psi_{x_{n}}^{p_{n}^{s}, p_{n}^{u}}$. This is similar to Claim 2 in the proof of [BO18, Lemma 4.7] and [BO18, Proposition 4.8], except that we must use $\Sigma_{\epsilon}^{\star \#}$ instead of $\Sigma_{\epsilon}^{\#}$. The details are as follows.

Assume without loss of generality that $u_{n_{k}}=u_{0}$, for all $k \geq 0$, and $n_{k} \uparrow \infty$. Consider the chain $\underline{w} \in \Sigma^{\star} \in\left[u_{0}\right]$ which is given by the $\star-\epsilon$-relevance of $u_{0}$ such that $\pi(\underline{w}) \in$ $\mathrm{RWT}_{\chi}^{\epsilon}$. Consider the chains

$$
\underline{u}^{(l)} \in \Sigma^{\star}, l \geq 0, \text { where } u_{i}^{(l)}= \begin{cases}u_{i}, & \text { for } i \leq n_{l}, \\ w_{i-n_{l}}, & \text { for } i \geq n_{l} .\end{cases}
$$

Write $z_{l}:=\pi\left(\underline{u}^{(l)}\right)$. In [BO18, Lemma 4.7], the author uses [BO18, Lemma 4.5] in order to show that $\sup _{l \geq 0} \sup _{\xi \in T_{z_{l}} V^{s}\left(\underline{u}^{(l)}\right),|\xi|=1} S\left(z_{l}, \xi\right)<\infty$; Corollary 4.3 takes the place of [BO18, Lemma 4.5] in this argument.

With this is mind, [BO18, Lemma 4.7 (Claim 2)] can be carried out verbatim, and so there exists a linear invertible map $\pi_{x_{0}}^{s}: T_{z} V^{s}(\underline{u}) \rightarrow H^{s}\left(x_{0}\right)$ such that $\left\|\pi_{x_{0}}^{s}\right\|,\left\|\left(\pi_{x_{0}}^{s}\right)^{-1}\right\| \leq$ $e^{2 Q_{\epsilon}\left(x_{0}\right)^{\beta / 4}}$, and such that

$$
\text { for all } \xi \in T_{z} V^{s}(\underline{u}), \quad|\xi|=1, \quad S(z, \xi)=e^{ \pm \sqrt{\epsilon}} S\left(x_{0}, \pi_{x_{0}}^{s} \xi\right) .
$$

A similar statement holds for $\pi_{x_{0}}^{u}: T_{z} V^{u}(\underline{u}) \rightarrow H^{u}\left(x_{0}\right)$ and $U(\cdot, \cdot) . \pi_{x_{0}}^{s}$ and $\pi_{x_{0}}^{u}$ extend to the invertible linear map $\pi_{x_{0}}: T_{z} M \rightarrow T_{x_{0}} M$ by $\pi_{x_{0}} \mid T_{z} V^{s}(\underline{u})=\pi_{x_{0}}^{s}$ and $\pi_{x_{0}} \mid T_{z} V^{u}(\underline{u})=\pi_{x_{0}}^{u}$.

It then follows from the proof of [BO18, Proposition 4.8] that $\left\|\pi_{x_{0}}\right\|,\left\|\pi_{x_{0}}^{-1}\right\| \leq e^{2 \sqrt{\epsilon}}$. Thus, by Corollary 3.2, $\left\|C_{\chi}^{-1}(z)\right\| /\left\|C_{\chi}^{-1}\left(x_{0}\right)\right\|=e^{ \pm 3 \sqrt{\epsilon}}$. Similarly, by considering the shifted sequence, $\left\|C_{\chi}^{-1}\left(f^{n}(z)\right)\right\| /\left\|C_{\chi}^{-1}\left(x_{n}\right)\right\|=e^{ \pm 3 \sqrt{\epsilon}}$, for all $n \in \mathbb{Z}$.

Step 4. We can now continue to show recurrent $\epsilon$-weak temperability. Define $q:\left\{f^{n}(z)\right\}_{n \in \mathbb{Z}} \rightarrow\left\{e^{-\ell \epsilon / 3}\right\}_{\ell \in \mathbb{N}}$ by $q\left(f^{n}(z)\right):=b_{\epsilon} \cdot p_{n}^{s} \wedge p_{n}^{u}, \quad$ where $\quad b_{\epsilon}:=\max \{t \in$ 
$\left.\left\{e^{-\ell \epsilon / 3}\right\}_{\ell \in \mathbb{N}}: t \leq e^{-300 \sqrt{\epsilon} / \beta}\right\}$. By the definition of $\mathcal{V}_{\epsilon}^{\star}, p_{n}^{s} \wedge p_{n}^{u} \in\left\{e^{-\ell \epsilon / 3}\right\}_{\ell \in \mathbb{N}}$, for all $n \in \mathbb{Z}$; thus, since $\left\{e^{-\ell \epsilon / 3}\right\}_{\ell \in \mathbb{N}}$ is closed under multiplication, the definition of $q$ is proper. $q$ satisfies the assumptions of recurrent $\epsilon$-weak temperability as follows.

(1) By the definition of ' $\rightarrow \epsilon$ ' (recall $\S 3.1$ step (3)(c)), $q \circ f / q=e^{ \pm \epsilon}$.

(2) Since $\underline{u} \in \stackrel{\star}{\Sigma}_{\epsilon}^{\#}$, lim $\sup _{n \rightarrow \pm \infty} q \circ f^{n}(x)>0$.

(3) By the definition of double Pesin charts, $p_{n}^{s} \wedge p_{n}^{u} \leq Q_{\epsilon}\left(x_{n}\right)$, for all $n \in \mathbb{Z}$. Thus, for all $n \in \mathbb{Z}$ (recall Definition 2.2 for the definition of $Q_{\epsilon}$ ),

$$
\begin{aligned}
p_{n}^{s} \wedge p_{n}^{u} \leq Q_{\epsilon}\left(x_{n}\right) & \leq \frac{\epsilon^{90 / \beta}}{3^{6 / \beta}}\left\|C_{\chi}^{-1}\left(x_{n}\right)\right\|^{-48 / \beta} \\
& \leq \frac{\epsilon^{90 / \beta}}{3^{6 / \beta}}\left(e^{-3 \sqrt{\epsilon}}\left\|C_{\chi}^{-1}\left(f^{n}(z)\right)\right\|\right)^{-48 / \beta} \\
& \leq e^{3 \sqrt{\epsilon} \cdot 48 / \beta} \cdot Q_{\epsilon}\left(f^{n}(z)\right) \cdot e^{\epsilon / 3} \\
& \leq e^{300 \sqrt{\epsilon} / \beta} Q_{\epsilon}\left(f^{n}(z)\right) .
\end{aligned}
$$

Therefore $\quad q\left(f^{n}(z)\right) \leq e^{-300 \sqrt{\epsilon} / \beta} \cdot p_{n}^{s} \wedge p_{n}^{u} \leq e^{-300 \sqrt{\epsilon} / \beta} \cdot e^{300 \sqrt{\epsilon} / \beta} Q_{\epsilon}\left(f^{n}(z)\right) \leq$ $Q_{\epsilon}\left(f^{n}(z)\right)$, for all $n \in \mathbb{Z}$.

It follows that $\pi(\underline{u}) \in \mathrm{RWT}_{\chi}^{\epsilon}$. Thus, $\pi\left[\stackrel{\Sigma}{\Sigma}_{\epsilon}^{\#}\right] \subseteq \mathrm{RWT}_{\chi}^{\epsilon}$, and together with step $1, \pi\left[\stackrel{\Sigma}{\Sigma}_{\epsilon}^{\#}\right]=$ $\mathrm{RWT}_{\chi}^{\epsilon}$.

Definition 4.6. For all $u \in \stackrel{\mathcal{V}}{\epsilon}_{\epsilon}, \stackrel{\star}{Z}_{\epsilon}(u):=\pi\left[[u] \cap \stackrel{\star}{\Sigma}_{\epsilon}^{\#}\right] . \stackrel{\mathcal{Z}}{\epsilon}_{\epsilon}:=\left\{\stackrel{\star}{Z}_{\epsilon}(u): u \in \stackrel{\mathcal{V}}{\epsilon}_{\epsilon}\right\}$ is a cover of $\pi\left[\stackrel{\Sigma}{\Sigma}_{\epsilon}^{\#}\right]$.

COROLlary 4.7. (Local finiteness of $\stackrel{\star}{\mathcal{Z}}_{\epsilon}$ ) For all $\epsilon \in\left(0, \stackrel{\star}{\epsilon}_{\chi}\right]$, for all $u \in \stackrel{\mathcal{V}}{\epsilon}_{\epsilon}$, $\left\{v \in \stackrel{\star}{\mathcal{V}}_{\epsilon}\right.$ : $\left.\stackrel{\star}{Z}_{\epsilon}(u) \cap \stackrel{\star}{Z}_{\epsilon}(v) \neq \varnothing\right\}<\infty$.

This is the content of [BO18, Theorem 5.2] (and similarly [Sar13, Theorem 10.2] when $d=2$ ), where (as in step 3 in Theorem 4.5) Corollary 4.3 replaces [BO18, Lemma 4.5] in the proof of [BO18, Lemma 4.7]; and the rest of the inverse problem [BO18, §4] can be carried out verbatim with $\Sigma_{\epsilon}^{\star}$ replacing $\Sigma_{\epsilon}^{\#}$.

Assume that there exists a periodic point $p \in \chi$-hyp, and let $\epsilon \in\left(0, \stackrel{\star}{\epsilon}_{\chi}\right]$.

THEOREM 4.8. There exists a countable partition $\stackrel{\star}{\mathcal{R}}_{\epsilon}$ of $\bigcup_{v \in \stackrel{\mathcal{V}}{\epsilon}^{\star}} \stackrel{\star}{Z}_{\epsilon}(v)=\pi\left[\stackrel{\star}{\Sigma}_{\epsilon}^{\#}\right]$ such that the following statements hold.

(1) $\stackrel{\star}{\mathcal{R}}_{\epsilon}$ is a refinement of $\stackrel{\star}{\mathcal{Z}}_{\epsilon}$ : for all $Z \in \stackrel{\star}{\mathcal{Z}}_{\epsilon}, R \in \stackrel{\star}{\mathcal{R}}_{\epsilon}, R \cap Z \neq \varnothing \Rightarrow R \subseteq Z$.

(2) For all $v \in \stackrel{\mathcal{V}}{\epsilon}_{\epsilon}, \#\left\{R \in \stackrel{\mathcal{R}}{\epsilon}_{\epsilon}: R \subseteq \stackrel{\star}{Z}_{\epsilon}(v)\right\}<\infty$ [Sar13, §11].

(3) Symbolic local product structure. For all $R \in \stackrel{\mathcal{R}}{\epsilon}_{\epsilon}$, for all $x, y \in R$, there exists $z:=[x, y]_{R} \in R$, such that for all $i \geq 0, R\left(f^{i}(z)\right)=R\left(f^{i}(y)\right), \quad R\left(f^{-i}(z)\right)=$ $R\left(f^{-i}(x)\right)$, where $R(t)$ is the unique partition member of $\stackrel{\star}{\mathcal{R}}_{\epsilon}$ containing $t$, for $t \in \pi\left[\Sigma_{\epsilon}^{\star}\right]$. 
Given a locally finite cover as $\mathcal{Z}_{\epsilon}$ (see Corollary 4.7 ), such a refining partition as $\stackrel{\star}{\mathcal{R}}_{\epsilon}$ exists by the Bowen-Sinai refinement; see [Sar13, §11.1].

Definition 4.9. (1) $\stackrel{\star}{\star}_{\epsilon}:=\left\{\underline{R} \in \stackrel{\mathcal{R}}{\epsilon}^{\mathbb{Z}}: R_{i} \cap f^{-1}\left[R_{i+1}\right] \neq \varnothing\right.$, for all $\left.i \in \mathbb{Z}\right\}$.

(2) $\widehat{\Sigma}_{\epsilon}^{\#}:=\left\{\underline{R} \in \widehat{\Sigma}_{\epsilon}\right.$ : there exists $n_{k}, m_{k} \uparrow \infty$ such that $R_{n_{k}}=R_{n_{0}}, R_{-m_{k}}=R_{-m_{0}}$, for all $k \geq 0\}$.

(3) Every pair of partition members $R, S \in \mathcal{R}_{\epsilon}$ is said to be $\epsilon$-affiliated if there exists $u, v \in \stackrel{\mathcal{V}}{\epsilon}_{\epsilon}$ such that $R \subseteq \stackrel{\star}{Z}_{\epsilon}(u), S \subseteq \stackrel{\star}{Z}_{\epsilon}(v)$ and $\stackrel{\hbar}{Z}_{\epsilon}(u) \cap \stackrel{\star}{Z}_{\epsilon}(v) \neq \varnothing$ (see [Sar13, $\S 12.3])$.

Remark. By Corollary 4.7 and Theorem 4.8 , every partition member of $\stackrel{\star}{\mathcal{R}}_{\epsilon}$ has only a finite number of partition members $\epsilon$-affiliated to it.

THEOREM 4.10. Given $\stackrel{\star}{\widehat{\Sigma}}_{\epsilon}$ from Definition 4.9, there exists a factor map $\widehat{\pi}: \stackrel{\star}{\Sigma}_{\epsilon} \rightarrow M$ such that the following statements hold.

(1) $\widehat{\pi}$ is Hölder continuous with respect to the metric $d(\underline{R}, \underline{S})=\exp \left(-\min \left\{i \geq 0: R_{i} \neq\right.\right.$ $S_{i}$ or $\left.\left.R_{-i} \neq S_{-i}\right\}\right)$.

(2) $f \circ \widehat{\pi}=\widehat{\pi} \circ \sigma$, where $\sigma$ denotes the left-shift on $\widehat{\Sigma}_{\epsilon}$.

(3) $\left.\widehat{\pi}\right|_{\widehat{\Sigma}_{\epsilon}^{\#}}$ is finite-to-one.

(4) For all $\underline{R} \in \widehat{\widehat{\Sigma}}_{\epsilon}^{\star}, \widehat{\pi}(\underline{R}) \in \overline{R_{0}}$.

(5) $\widehat{\pi}\left[\widehat{\widehat{\Sigma}}_{\epsilon}^{\#}\right]$ carries all $\chi$-hyperbolic invariant probability measures.

This theorem is the content of [Sar13, Theorem 1.3] (and [BO18, Theorem 1.1]).

Proposition 4.11. For all $\epsilon \in\left(0, \stackrel{\star}{\epsilon}_{\chi}\right]$,

$$
\widehat{\pi}\left[\stackrel{\star}{\widehat{\Sigma}_{\epsilon}^{\#}}\right]=\pi\left[\stackrel{\star}{\Sigma}_{\epsilon}^{\#}\right]=\bigcup \stackrel{\star}{\mathcal{R}}_{\epsilon} .
$$

Proof. $\pi\left[\stackrel{\star}{\Sigma}_{\epsilon}^{\#}\right]=\bigcup \stackrel{\star}{\mathcal{R}}_{\epsilon}$ by definition, since $\stackrel{\star}{\mathcal{R}}_{\epsilon}$ is a partition of $\pi\left[\stackrel{\star}{\Sigma}_{\epsilon}^{\#}\right]$. We need to show that $\widehat{\pi}\left[\widehat{\Sigma}_{\epsilon}^{\#}\right]=\pi\left[\stackrel{\Sigma}{\Sigma}_{\epsilon}^{\#}\right]$.

(). Let $\underline{u} \in \stackrel{\star}{\Sigma}_{\epsilon}^{\#}, \pi(\underline{u}) \in \stackrel{\star}{\mathcal{R}}_{\epsilon}$. Write $R_{i}:=$ unique element of $\stackrel{\star}{\mathcal{R}}_{\epsilon}$ which contains $\pi\left(\sigma^{i} \underline{u}\right)=f^{i}(\pi(\underline{u})), i \in \mathbb{Z}$. It follows that $\underline{R}:=\left(R_{i}\right)_{i \in \mathbb{Z}} \in \widehat{\widehat{\Sigma}}_{\epsilon}$, and that $\widehat{\pi}(\underline{R})=\pi(\underline{u})$ by the uniqueness of a shadowed orbit (by [B018, Proposition 3.12, Proposition 3.5] $\{\widehat{\pi}(\underline{R})\}=V^{s}(\underline{u}) \pitchfork V^{u}(\underline{u})$, and by Claim 3.7, $\left.\widehat{\pi}(\underline{R})=\pi(\underline{u})\right)$. Then by definition, for all $i \in \mathbb{Z}, R_{i} \subseteq \stackrel{\star}{Z}_{\epsilon}\left(u_{i}\right)$. Since $\underline{u} \in \stackrel{\star}{\Sigma}_{\epsilon}^{\#}$, there exists $i_{k} \uparrow \infty$ such that $u_{i_{k}}=\tilde{u}$. By Theorem 4.8(2), $Z(\widetilde{u})$ contains finitely many elements of $\mathcal{R}_{\epsilon}$. So, by the pigeonhole principle, $\underline{R} \in \widehat{\Sigma}_{\epsilon}^{\star}$ (see Theorem 4.8).

(C). Let $R, S \in \stackrel{\star}{\mathcal{R}}_{\epsilon}$ such that there exists $x \in R \cap f^{-1}[S]$. Let $u \in \stackrel{\mathcal{V}}{\epsilon}_{\epsilon}$ such that $R \subseteq$ $\stackrel{\star}{Z}_{\epsilon}(u)$. Then there exists $\underline{u} \in \stackrel{\Sigma}{\Sigma}_{\epsilon}^{\#} \cap[u]$ such that $\pi(\underline{u})=x$, and so $S \subseteq \stackrel{Z}{Z}_{\epsilon}\left(u_{1}\right)$. Given 
a chain $\underline{R} \in \stackrel{\widehat{\Sigma}}{\star}_{\epsilon}^{\#}$, choose $\stackrel{\hbar}{Z}_{\epsilon}\left(u_{0}\right) \supseteq R_{0}$, and construct this way a chain $\underline{u} \in \stackrel{\Sigma}{\Sigma}_{\epsilon}$ such that $R_{i} \subseteq \stackrel{\star}{Z}_{\epsilon}\left(u_{i}\right)$ for all $i \in \mathbb{Z}$. By the uniqueness of a shadowed orbit, $\pi(\underline{u})=\widehat{\pi}(\underline{R})$. By Corollary 4.7, and the pigeonhole principle, $\underline{u} \in \stackrel{\star}{\Sigma}_{\epsilon}^{\#}$.

COROLLARY 4.12. Let $p$ be a $\chi$-hyperbolic periodic point, such that $\mathrm{RWT}_{\chi}^{\stackrel{\star}{\epsilon}_{\chi}} \neq \varnothing$. Then for all $\epsilon \in\left(0, \stackrel{\star}{\epsilon}_{\chi}\right]$

$$
\widehat{\pi}\left[\widehat{\Sigma}_{\epsilon}^{\#}\right]=\pi\left[\stackrel{\star}{\Sigma}_{\epsilon}^{\#}\right]=\mathrm{RWT}_{\chi}^{\epsilon} .
$$

Proof. In Theorem 4.5 we saw that $\pi\left[\stackrel{\Sigma}{\star}_{\epsilon}^{\#}\right]=\mathrm{RWT}_{\chi}^{\epsilon}$. In Proposition 4.11 we showed the equality $\widehat{\pi}\left[\widehat{\Sigma}_{\epsilon}^{\#}\right]=\pi\left[\stackrel{\Sigma}{\Sigma}_{\epsilon}^{\#}\right]$. Therefore we are done.

Definition 4.13. $\operatorname{RWT}_{\chi}^{\star}:=\mathrm{RWT}_{\chi}^{\stackrel{\iota^{\epsilon}}{\epsilon_{\chi}}}$ is called the set of recurrently codable points.

Notice that, by Claim 2.6, $\bigcup_{0<\epsilon \leq 2 / 3 \epsilon_{\chi}^{\star}} \operatorname{RWT}_{\chi}^{\epsilon} \subseteq \mathrm{RWT}_{\chi}^{\star}$.

5. Ergodic homoclinic classes, maximal irreducible components and coding infinite conservative measures

The aim of this section is to construct an irreducible coding of an ergodic homoclinic class, which lifts all conservative (possibly infinite) measures on it (see definition below), as an extension to the preceding result in [BCS], which only treats probability measures.

In this section $\epsilon$ is fixed and equals $\stackrel{\star}{\epsilon}_{\chi}$. The $\epsilon$ subscript on $\stackrel{\star}{\Sigma}_{\epsilon}, \stackrel{\star}{\Sigma}_{\epsilon}^{\#}, \stackrel{\mathcal{R}}{\epsilon}_{\epsilon}, \stackrel{\Sigma}{\Sigma}_{\epsilon}, \stackrel{\star}{\Sigma}_{\epsilon}^{\#}, \stackrel{\mathcal{V}}{\epsilon}_{\epsilon}, \stackrel{\mathcal{Z}}{\mathcal{Z}}_{\epsilon}$ will be omitted to ease notation.

Let $p$ be a periodic point in $\chi$-summ. Since $p$ is periodic, $\left\|C_{\chi}^{-1}(\cdot)\right\|$ is bounded along the orbit of $p$, and therefore $p \in \mathrm{RWT}_{\chi}^{\star}$. Every point $x \in \mathrm{RWT}_{\chi}^{\star}$ is (recurrently) codable, and so has a local stable manifold $V^{s}(x)$ (e.g. $\left.V^{s}(\underline{u}), \underline{u} \in \pi^{-1}[\{x\}] \cap \Sigma^{\star}\right)$, and a global stable manifold $W^{s}(x):=\bigcup_{n \geq 0} f^{-n}\left[V^{s}\left(f^{n}(x)\right)\right]$ (similarly for a global unstable manifold).

Definition 5.1. A (possibly infinite) measure-preserving transformation, $(X, \mathcal{B}, \mu, T)$, is called conservative if for every set $A \in \mathcal{B}$ such that $\mu(A)>0$, there exists $n>0$ such that $\mu\left(T^{-n}[A] \cap A\right)>0$.

By Halmos's recurrence theorem (see [Aar97, §1.1.1]), a measure-preserving transformation is conservative if and only if it satisfies the statement of the Poincaré recurrence theorem:

for all $A \in \mathcal{B}, \mu\left(A \backslash\left\{x \in A\right.\right.$ : there exists $n_{k}, m_{k} \uparrow \infty$ such that

$$
\left.\left.f^{n_{k}}(x), f^{-m_{k}}(x) \in A \text {, for all } k \geq 0\right\}\right)=0 .
$$

Definition 5.2. The $\chi$-ergodic homoclinic class of $p$ is

$$
H_{\chi}(p):=\left\{x \in \operatorname{RWT}_{\chi}^{\star}: W^{u}(x) \pitchfork W^{s}(o(p)) \neq \varnothing, W^{s}(x) \pitchfork W^{u}(o(p)) \neq \varnothing\right\},
$$


where $\pitchfork$ denotes transverse intersections of full codimension, $o(p)$ is the (finite) orbit of $p$, and $W^{s}(\cdot), W^{u}(\cdot)$ are the global stable and unstable manifolds of a point (or points in an orbit), respectively.

This notion was introduced in [RHRHTU11], with a set of Lyapunov regular points replacing $\mathrm{RWT}_{\chi}^{\star}$. Every ergodic conservative $\chi$-hyperbolic measure is carried by a $\chi$-ergodic homoclinic class of some periodic hyperbolic point.

Remark. Notice that $H_{\chi}(p) \subset \overline{W^{s}(o(p)) \pitchfork W^{u}(o(p))} \equiv$ Newhouse's definition of a homoclinic class [New72]; in particular, $H_{\chi}(p)$ is not necessarily closed.

Definition 5.3.

(1) Define the relation $\sim \subseteq \stackrel{\star}{\mathcal{R}} \times \stackrel{\star}{\mathcal{R}}$ by $R \sim S \Longleftrightarrow$ there exist $n_{R S}, n_{S R} \in \mathbb{N}$ such that $R \stackrel{n_{R S}}{\longrightarrow} S, S \stackrel{n_{S R}}{\longrightarrow} R$, that is, there exists $\left\{R_{i}\right\}_{i=0}^{n_{S R}+n_{R S}}$ such that $R_{0}=R, R_{n_{R S}}=$ $S, R_{n_{S R}+n_{R S}}=R$ and $f^{-1}\left[R_{i}\right] \cap R_{i-1} \neq \varnothing$ for all $1 \leq i \leq n_{R S}+n_{S R}$. The relation $\sim$ is transitive and symmetric. When restricted to $\{R \in \stackrel{\star}{\mathcal{R}}: R \sim R\}$, it is also reflexive, and thus an equivalence relation. Denote the corresponding equivalence class of some representative $R \in \stackrel{\star}{\mathcal{R}}, R \sim R$ by $\langle R\rangle$.

(2) A maximal irreducible component in $\stackrel{\star}{\widehat{\Sigma}}$, corresponding to $R \in \stackrel{\star}{\mathcal{R}}$ such that $R \sim R$, is $\left\{\underline{R} \in \hat{\widehat{\Sigma}}: \underline{R} \in\langle R\rangle^{\mathbb{Z}}\right\}$.

LEMMA 5.4. Let $p \in \chi$-summ such that there exists $l \in \mathbb{N}$ such that if $f^{l}(p)=p$, then $p \in \chi$-hyp.

Proof. We prove an exponential contraction strictly stronger than $e^{-\chi}$ on $H^{s}(p)$. The case for $H^{u}(p)$ is similar. First assume that $f(p)=p$. Since $p$ is $\chi$-summable, for all $\xi \in$ $H^{s}(p)$ with $|\xi|=1, \sum_{m=0}^{\infty}\left|d_{p} f^{m} \xi\right|^{2} e^{2 \chi m}<\infty$. Let $\left\{\xi_{i}\right\}_{i=1}^{s(p)}$ be an orthonormal basis for $H^{s}(p)$ (with respect to $\langle\cdot, \cdot\rangle_{p}$, the Riemannian form at $T_{p} M$ ). For all $m \geq 0$, there exists $\xi^{(m)} \in H^{s}(p)$ with $\left|\xi^{(m)}\right|=1$ such that $\left|d_{p} f^{m} \xi^{(m)}\right|=\left\|\left.d_{p} f^{m}\right|_{H^{s}(p)}\right\|$. Therefore, for $a_{i}^{(m)}:=\left\langle\xi^{(m)}, \xi_{i}\right\rangle_{p}$

$$
\begin{aligned}
& \sum_{m=0}^{\infty}\left\|\left.d_{p} f^{m}\right|_{H^{s}(p)}\right\|^{2} e^{2 \chi m}=\sum_{m=0}^{\infty}\left|d_{p} f^{m} \xi^{(m)}\right|^{2} e^{2 \chi m} \leq \sum_{m=0}^{\infty}\left(\sum_{i=1}^{s(p)}\left|a_{i}^{(m)}\right| \cdot\left|d_{p} f^{m} \xi_{i}\right|\right)^{2} e^{2 \chi m} \\
& \leq \sum_{m=0}^{\infty}\left(\sum_{i=1}^{s(p)}\left|d_{p} f^{m} \xi_{i}\right|\right)^{2} e^{2 \chi m}=\sum_{i, j=1}^{s(p)} \sum_{m=0}^{\infty}\left(\left|d_{p} f^{m} \xi_{i}\right| e^{\chi m}\right)\left(\left|d_{p} f^{m} \xi_{j}\right| e^{\chi^{m}}\right) \\
& \quad \leq \sum_{i, j=1}^{s(p)} \sqrt{\sum_{m=0}^{\infty}\left|d_{p} f^{m} \xi_{i}\right|^{2} e^{2 \chi m}} \cdot \sqrt{\left.\sum_{m=0}^{\infty}\left|d_{p} f^{m} \xi_{j}\right|^{2} e^{2 \chi m} \mid \leq 1\right)} \quad(\because \text { Cauchy-Schwarz }) \\
& \leq d^{2} \max _{i \leq s(p)}\left\{\sum_{m=0}^{\infty}\left|d_{p} f^{m} \xi_{i}\right|^{2} e^{2 \chi m}\right\}<\infty .
\end{aligned}
$$


The derivative $d_{p} f: H^{s}(p) \rightarrow H^{s}(p)$ is a linear map. Working in coordinates, it is sufficient to assume without loss of generality that $d_{p} f$ is a Jordan block $J_{s(p)}(\lambda)$ of dimension $s(p)$, with eigenvalue $\lambda$ for some $\lambda \in \mathbb{R} \backslash\{0\}$ (since $f$ is a diffeomorphism).

Write $J_{S(p)}=\lambda I+N$ where $N$ denotes the nilpotent matrix whose entries right above the diagonal are all $1 \mathrm{~s}$ and all other entries are $0 \mathrm{~s}$, and $I$ denotes the identity matrix. Then $N^{s(p)}=0$. By the binomial theorem, if $m \geq s(p)$, then

$$
J_{s(p)}(\lambda)^{m}=(\lambda I+N)^{m}=\sum_{k=0}^{s(p)}\left(\begin{array}{l}
m \\
k
\end{array}\right) \lambda^{m-k} N^{k} .
$$

Since $J_{S(p)}(\lambda)$ is a Jordan block, it admits an eigenvector with an eigenvalue $\lambda$, and so $\left\|J_{s(p)}(\lambda)\right\| \geq|\lambda|$, and $\left\|\left.d_{p} f^{m}\right|_{H^{s}(p)}\right\| \geq|\lambda|^{m}$. Hence, by equation (3), $\sum_{m=0}^{\infty}|\lambda|^{2 m} e^{2 \chi m} \leq$ $\sum_{m=0}^{\infty}\left\|\left.d_{p} f^{m}\right|_{H^{s}(p)}\right\|^{2} e^{2 \chi m}<\infty$, and so $0<|\lambda|<e^{-\chi}$.

On the other hand, by equation (4), $\left\|\left.d_{p} f^{m}\right|_{H^{s}(p)}\right\| \leq C \cdot m^{s(p)} \cdot|\lambda|^{m}$ for some $C=C(p)>0$. Hence, $\left\|\left.d_{p} f^{m}\right|_{H^{s}(p)}\right\| \leq C \cdot m^{s(p)} \cdot e^{\chi^{\prime} m}$, where $\chi^{\prime}:=\log |\lambda|<-\chi$. Thus, $\lim \sup _{m \rightarrow \infty}(1 / m) \log \left\|\left.d_{p} f^{m}\right|_{H^{s}(p)}\right\|<-\chi$. This concludes the proof for the case $f(p)=p$.

In the case where the period of $p$ is $l>1$, write, for all $\xi \in H^{s}(p)$,

$$
\sum_{m=0}^{\infty}\left|d_{p} f^{m} \xi\right|^{2} e^{2 \chi m}=\sum_{j=0}^{l-1} e^{2 j \chi} \sum_{m=0}^{\infty}\left|d_{f^{j}(p)}\left(f^{l}\right)^{m}\left(d_{p} f^{j} \xi\right)\right|^{2} e^{2(l \chi) m}<\infty .
$$

Then by the first part of this proof, there exist $\lambda_{j}, j=0, \ldots, l-1$, such that $0<\left|\lambda_{j}\right|<$ $e^{-\chi \cdot l}$, and $C_{j}=C_{j}(p)>0$ such that for all $0 \leq j \leq l-1, l \geq 0,\left\|\left.d_{p} f^{m \cdot l+j}\right|_{H^{s}(p)}\right\| \leq$ $\max _{i}\left\{C_{i}\right\} \max _{j}\left\{\left|\lambda_{j}\right|^{m}\right\} \cdot m^{s(p)}$. As in the case of $f(p)=p$, this is sufficient.

Definition 5.5. For all $x \in \mathrm{WT}_{\chi}^{\star}$,

$$
\begin{aligned}
& p_{n}^{u}(x):=\max \left\{t \in\left\{e^{-\ell \epsilon / 3}\right\}_{\ell \in \mathbb{N}}: e^{-\epsilon N} t \leq Q_{\epsilon}\left(f^{n-N}(x)\right), \text { for all } N \geq 0\right\}, \\
& p_{n}^{s}(x):=\max \left\{t \in\left\{e^{-\ell \epsilon / 3}\right\}_{\ell \in \mathbb{N}}: e^{-\epsilon N} t \leq Q_{\epsilon}\left(f^{n+N}(x)\right), \text { for all } N \geq 0\right\} .
\end{aligned}
$$

This definition was introduced in [Sar13, Lemma 4.6], and is due to Ledrappier. The maximum is over a non-empty set because of $\epsilon$-weak temperability.

Note that, for all $n \in \mathbb{Z}, \psi_{f^{n}(x)}^{p^{s}(x), p_{n}^{u}(x)} \rightarrow \rightarrow_{\epsilon} \psi_{f^{n+1}(x)}^{p^{s}(x), p_{n+1}^{u}(x)}$.

LEMMA 5.6. Let $p$ be a $\chi$-hyperbolic periodic point, and let $\underline{u}:=\left(\psi_{f_{n}^{n}(p)}^{p^{s}(p), p_{n}^{u}(p)}\right)_{n \in \mathbb{Z} . \text { Let }}$ $x \in V^{u}(\underline{u}) \cap \mathrm{RWT}_{\chi}^{\star}$ such that $\operatorname{dim} H^{s}(x)=\operatorname{dim} H^{s}(p)$. Then

$\limsup _{n \rightarrow \infty} \sup _{\xi_{n} \in H^{s}\left(f^{-n}(x)\right),\left|\xi_{n}\right|=1} S\left(f^{-n}(x), \xi_{n}\right) \leq \max _{i}\left\{\sup _{\eta_{i} \in H^{s}\left(f^{i}(p)\right),\left|\eta_{i}\right|=1} S\left(f^{i}(p), \eta_{i}\right)\right\}<\infty$.

A similar claim holds for $x \in V^{s}(\underline{u}) \cap \mathrm{RWT}_{\chi}^{\star}$, but with $n \rightarrow-\infty$.

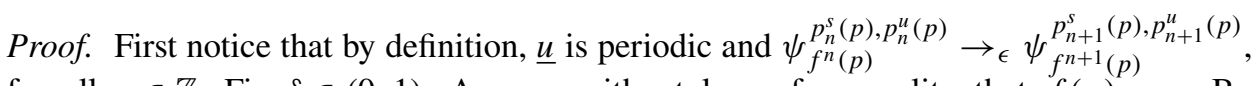
for all $n \in \mathbb{Z}$. Fix $\delta \in(0,1)$. Assume without loss of generality that $f(p)=p$. By 
Definition 5.5, if $f(p)=p$, then $\sigma \underline{u}=\underline{u}$. By the inclination lemma [BS02, Theorem 5.7.2], we may assume without loss of generality that $d_{C^{1}}\left(V^{s}\left(f^{-i}(x)\right), V^{s}(\underline{u})\right) \leq \tau$ for all $i \geq 0$, where $\tau>0$ is arbitrarily small, $V^{s}\left(f^{-i}(x)\right)$ is the part of $W^{s}\left(f^{-i}(x)\right)$ which is close in $C^{1}$-norm to $V^{s}(\underline{u})$, and the $C^{1}$-distance is calculated in the chart $\psi_{p}^{Q_{\epsilon}(p)}$.

In particular, since $V^{s}(\underline{u})$ is an admissible manifold in $\psi_{p}^{Q_{\epsilon}(p)}, V^{s}\left(f^{-i}(x)\right)$ is the graph of a $C^{1}$-smooth function. Denote the function representing the graph of $V^{s}(\underline{u})$ by $F$, and the function representing the graph of $V^{s}\left(f^{-i}(x)\right)$ by $G_{i}, i \geq 0$.

Let $P_{s}: \mathbb{R}^{d} \rightarrow \mathbb{R}^{s(x)}$ be the projection to the $s(x)$ first coordinates, and let $\xi \in H^{s}(x)$ such that $\left|d_{x}\left(f^{-1}\right) \xi\right|=1 . \quad \xi=d_{\psi_{p}^{-1}(x)} \psi_{p}\left(u, d_{P_{s} \psi_{p}^{-1}(x)} G_{0} u\right)$ for some $u \in \mathbb{R}^{s(p)}$. Define $\eta=\eta(\xi):=d_{0} \psi_{p}\left(u, d_{0} F u\right) \in H^{s}(p)$. Write again $d_{x}\left(f^{-1}\right) \xi=$ $d_{\psi_{p}^{-1}\left(f^{-1}(x)\right)} \psi_{p}\left(v, d_{P_{s} \psi_{p}^{-1}\left(f^{-1}(x)\right)} G_{1} v\right)$ for some $v \in \mathbb{R}^{s(x)}$, and define $\zeta=\zeta(\xi):=$ $d_{0} \psi_{p}\left(v, d_{0} F v\right) \in H^{s}(p)$. Notice that $\eta: T_{x} V^{s}(x) \rightarrow H^{s}(p)$ is a linear map, and in fact the definition extends naturally to $\eta: T_{f^{-i}(x)} V^{s}\left(f^{-i}(x)\right) \rightarrow H^{s}(p)$, for all $i \geq 0$. Thus, by the inclination lemma and by choosing $\tau>0$ sufficiently small, we may assume without loss of generality that $\left.|| d_{x}\left(f^{-1}\right) \xi\right|^{2}-\left|d_{p}\left(f^{-1}\right) \eta\right|^{2} \mid \leq \delta$. Define $\rho:=\max \{S(x, \xi) / S(p, \eta), 1\}$.

Step 1: Refining the estimate from [Sar13, Lemma 7.2]. We have

$$
S^{2}\left(f^{-1}(x), d_{x}\left(f^{-1}\right) \xi\right) / S^{2}(p, \zeta) \leq \rho^{2} e^{-\delta}
$$

The proof is as follows:

$$
\begin{aligned}
S^{2}\left(f^{-1}(x), d_{x}\left(f^{-1}\right) \xi\right) & =2 \sum_{m=0}^{\infty}\left|d_{f^{-1}(x)} f^{m} d_{x}\left(f^{-1}\right) \xi\right|^{2} e^{2 \chi m} \\
& =S^{2}(x, \xi) e^{2 \chi}+2\left|d_{x}\left(f^{-1}\right) \xi\right|^{2} \\
& \leq \rho^{2} e^{2 \chi} S^{2}(p, \eta)+2\left|d_{x}\left(f^{-1}\right) \xi\right|^{2} . \\
S^{2}\left(p, d_{p}\left(f^{-1}\right) \eta\right) & =2 \sum_{m=0}^{\infty}\left|d_{p} f^{m} d_{p}\left(f^{-1}\right) \eta\right|^{2} e^{2 \chi m}=S^{2}(p, \eta) e^{2 \chi}+2\left|d_{p}\left(f^{-1}\right) \eta\right|^{2} .
\end{aligned}
$$

Then

$$
\begin{aligned}
& \frac{S^{2}\left(f^{-1}(x), d_{x}\left(f^{-1}\right) \xi\right)}{S^{2}\left(p, d_{p}\left(f^{-1}\right) \eta\right)} \\
& \leq \frac{\rho^{2} e^{2 \chi} S^{2}(p, \eta)+2\left|d_{x}\left(f^{-1}\right) \xi\right|^{2}}{S^{2}(p, \eta) e^{2 \chi}+2\left|d_{p}\left(f^{-1}\right) \eta\right|^{2}} \\
& =\rho^{2}-\frac{2\left(\rho^{2}-1\right)\left|d_{p}\left(f^{-1}\right) \eta\right|^{2}+2\left(\left|d_{p}\left(f^{-1}\right) \eta\right|^{2}-\left|d_{x}\left(f^{-1}\right) \xi\right|^{2}\right)}{S^{2}\left(p, d_{p}\left(f^{-1}\right) \eta\right)} .
\end{aligned}
$$

Now, if $\rho \geq e^{\sqrt{\delta}}$, then $\rho^{2}-1 \geq 2 \sqrt{\delta}$, and so $\left(\rho^{2}-1\right)\left|d_{p}\left(f^{-1}\right) \eta\right|^{2}+\left(\left|d_{p}\left(f^{-1}\right) \eta\right|^{2}-\right.$ $\left.\left|d_{x}\left(f^{-1}\right) \xi\right|^{2}\right) \geq\left(\rho^{2}-1\right)(1-\delta)-\delta \geq\left(\rho^{2}-1\right)(1-\delta)-\left(\rho^{2}-1\right) \delta / 2 \sqrt{\delta}=\left(\rho^{2}-1\right)$ $(1-\delta-\sqrt{\delta} / 2) \geq\left(\rho^{2}-1\right)(1-3 / 2 \sqrt{\delta}) \geq\left(\rho^{2}-1\right) e^{-2 \sqrt{\delta}}$, for small enough $\delta \in(0,1)$. 
We then get that, all together,

$$
\begin{aligned}
\frac{S^{2}\left(f^{-1}(x), d_{x}\left(f^{-1}\right) \xi\right)}{S^{2}\left(p, d_{p}\left(f^{-1}\right) \eta\right)} & \leq \rho^{2}-\frac{2\left(\rho^{2}-1\right) e^{-2 \sqrt{\delta}}}{S^{2}\left(p, d_{p}\left(f^{-1}\right) \eta\right)} \leq \rho^{2}-\frac{2\left(\rho^{2}-1\right) e^{-2 \sqrt{\delta}}}{\left\|C_{\chi}^{-1}(p)\right\| \cdot\left|d_{p}\left(f^{-1}\right) \eta\right|} \\
& \leq \rho^{2}-\frac{2\left(\rho^{2}-1\right) e^{-2 \sqrt{\delta}}}{\left\|C_{\chi}^{-1}(p)\right\|(1+\delta)} \leq \rho^{2}\left(1-\frac{2\left(1-1 / \rho^{2}\right) e^{-2 \sqrt{\delta}}}{\left\|C_{\chi}^{-1}(p)\right\| e^{\delta}}\right) \\
& \leq \rho^{2}\left(1-\frac{2\left(1-1 /\left(e^{2 \sqrt{\delta}}\right)\right) e^{-2 \sqrt{\delta}}}{\left\|C_{\chi}^{-1}(p)\right\| e^{\delta}}\right) \leq \rho^{2}\left(1-\frac{2 \sqrt{\delta} e^{-2 \sqrt{\delta}}}{\left\|C_{\chi}^{-1}(p)\right\| e^{\delta}}\right) .
\end{aligned}
$$

For $\delta \in(0,1)$ small enough so $\sqrt{\delta}^{-1 / 2} e^{-1} /\left\|C_{\chi}^{-1}(p)\right\| \geq 1$,

$$
\frac{S^{2}\left(f^{-1}(x), d_{x}\left(f^{-1}\right) \xi\right)}{S^{2}\left(p, d_{p}\left(f^{-1}\right) \eta\right)} \leq \rho^{2}(1-2 \delta) \leq \rho^{2} e^{-2 \delta} .
$$

Since both $\zeta$ and $\eta$ depend continuously on $\xi$, and can be made arbitrarily close with $\tau>0$ small enough, and since $S^{2}(p, \cdot)$ is continuous (see [BO18, Theorem 2.8]), we may assume without loss of generality that $S^{2}(p, \zeta) / S^{2}\left(p, d_{p}\left(f^{-1}\right) \eta\right) \in\left[e^{-\delta}, e^{\delta}\right]$. Thus we get in total that

$$
\frac{S^{2}\left(f^{-1}(x), d_{x}\left(f^{-1}\right) \xi\right)}{S^{2}(p, \zeta)} \leq \rho^{2} e^{-\delta} .
$$

Step 2: Estimating the limit. The estimates of step 1 hold for all $f^{-n}(x)$ for $n \geq 0$ large enough so $d_{C^{1}}\left(V^{s}\left(f^{-i}(x)\right), V^{s}(\underline{u})\right) \leq \tau$ for all $i \geq n$. Let $n_{\delta}:=\min \{n \geq 0$ : for all $i \geq$ $\left.n, d_{C^{1}}\left(V^{s}\left(f^{-i}(x)\right), V^{s}(\underline{u})\right) \leq \tau\right\}$, where $0<\tau=\tau(\delta)$ (thus step 1 treats all $f^{-n}(x)$ for all $n \geq n_{\delta}$ ). That means that the ratio in equation (6) is either bounded by $e^{\sqrt{\delta}}$, or it improves by a factor of at least $e^{\delta}$, with each iteration of $f^{-1}$ (starting from $f^{-n_{\delta}}(x)$ ). On the other hand, when $1 \leq \rho \leq e^{\sqrt{\delta}}$, by equation (5), with an iteration of $f^{-1}$, the bound may deteriorate to at most $e^{3 \sqrt{\delta}}$. Thus,

$$
\limsup _{n \rightarrow \infty} \frac{S^{2}\left(f^{-n}(x), d_{x}\left(f^{-n}\right) \xi\right)}{S^{2}\left(p, \eta\left(d_{x}\left(f^{-n}\right) \xi\right)\right)} \leq e^{3 \sqrt{\delta}} .
$$

Now, since $\delta>0$ was arbitrary, we get, for all $\xi \in H^{s}(x) \backslash\{0\}$, that

$$
\limsup _{n \rightarrow \infty} \frac{S^{2}\left(f^{-n}(x), d_{x}\left(f^{-n}\right) \xi\right)}{S^{2}\left(p, \eta\left(d_{x}\left(f^{-n}\right) \xi\right)\right)} \leq 1 .
$$

From that, since $\left.\left.\lim _{n \rightarrow \infty}\left|\eta\left(d_{x}\left(f^{-n}\right) \xi\right) /\right| d_{x}\left(f^{-n}\right) \xi\right) \mid\right) \mid=1$ (by the inclination lemma and the definition of $\left.\eta: T_{f^{-n}(x)} V^{s}\left(f^{-n}(x)\right) \rightarrow H^{s}(p)\right)$, the lemma follows.

The reason why we got a better result here than in [BO18, Lemma 4.6] and [Sar13, Lemma 7.2] is that here the centers of charts are $f^{-i}(p)$, and there is no distortion as a consequence of non-full overlap between $f^{-1}\left(x_{i}\right)$ and $x_{i-1}$.

Definition 5.7. For all $x \in \mathrm{RWT}_{\chi}^{\star}=\bigcup_{\star} \stackrel{\star}{\mathcal{R}}$, the itinerary of $x$ is $\underline{R}(x):=\left(R\left(f^{i}(x)\right)\right)_{i \in \mathbb{Z}}$, where $R\left(f^{i}(x)\right):=$ unique element of $\stackrel{\star}{\mathcal{R}}$ which contains $f^{i}(x)$. 
Notice that $\underline{R}(\cdot): \mathrm{RWT}_{\chi}^{\star} \rightarrow \stackrel{\star}{\widehat{\Sigma}}$ is one-to-one and is a measurable map such that $\underline{R} \circ$ $f=\sigma \circ \underline{R}$ and $\widehat{\pi} \circ \underline{R}=$ Id. Moreover, for all $x \in \mathrm{RWT}_{\chi}^{\star}, \underline{R}(x) \in \widehat{\Sigma}^{\star}$; see the proof of Proposition 4.11.

PROPOSITION 5.8. For every $\chi$-hyperbolic periodic point $p$, there exists $\widetilde{\Sigma} \subseteq \widehat{\Sigma}$, a maximal irreducible component, such that $\widehat{\pi}[\widetilde{\Sigma}] \supseteq H_{\chi}(p)$ modulo all conservative (possibly infinite) Borel measures which are carried by $H_{\chi}(p)$.

This is an adaptation of the proof by Buzzi, Crovisier and Sarig in [BCS, Lemma 3.11, Lemma 3.12]. They proved the proposition for finite invariant measures and in dimension 2. As stated in [BCS], the symbolic dynamics of [BO18] can be used to extend the proof to the higher-dimensional case, but still only for finite invariant measures. Here we explain how to extend the proof to cover also all conservative infinite measures.

Proof. Let $\mu$ be a conservative, invariant, (possibly infinite) Borel measure carried by $H_{\chi}(p)$. By Corollary 4.12 and Proposition 4.11, $H_{\chi}(p) \subseteq \widehat{\pi}\left[\widehat{\Sigma}^{\#}\right]=\bigcup \stackrel{\star}{\mathcal{R}}$. Since $\underline{R}(\cdot)$ is one-to-one, $\widehat{\mu}:=\mu \circ \underline{R}^{-1}$ is a well-defined invariant and conservative measure on $\widehat{\Sigma}^{\#}$, and $\widehat{\mu} \circ \widehat{\pi}^{-1}=\mu$. Thus $\widehat{\mu}$ is carried by

$$
\begin{array}{r}
\widehat{\widehat{\Sigma}}^{\# \#}:=\left\{\underline{R} \in \widehat{\widehat{\Sigma}}^{\star \#}: \text { there exists } a \in \stackrel{\mathcal{R}}{\mathcal{R}}^{\star} \text { such that } R_{i}=a\right. \text { for infinitely many } \\
\qquad i \geq 0 \text { and for infinitely many } i \leq 0\} .
\end{array}
$$

Therefore, $\widehat{\pi}\left[\widehat{\widehat{\Sigma}}^{\# \#}\right] \cap H_{\chi}(p)$ carries all conservative measures which are carried by $H_{\chi}(p)$. While chains in $\widehat{\Sigma}^{\star} \#$ are required to have a symbol which repeats infinitely often in the future and in the past, chains in $\widehat{\widehat{\Sigma}}^{\#}$ are allowed to have one symbol which repeats infinitely often in the future, and possibly a different symbol which repeats infinitely often in the past. Each chain $\underline{R}$ in $\widehat{\widehat{\Sigma}}^{\# \#}$ has the following form:

$$
\ldots a, \tilde{p}_{-i}, a, \tilde{p}_{-i+1}, a \ldots a, \tilde{p}_{-1}, a \ldots a, \tilde{p}_{1}, a \ldots a, \tilde{p}_{i-1}, a \ldots a, \tilde{p}_{i}, a \ldots
$$

where $\tilde{p}_{i}$ denotes a finite word which connects $a$ to $a$. For each word $w_{n}:=$ $a, \tilde{p}_{-n}, a, \tilde{p}_{-n+1}, a, \ldots, a, \tilde{p}_{n-1}, a, \tilde{p}_{n} a$, let $x_{\underline{R}}^{(n)}$ be the image of the periodic extension of $w_{n}$.

Step 0. Construction of a countable and dense collection of hyperbolic periodic points. For every chain $\underline{R}$ as in equation (8) such that $\widehat{\pi}(\underline{R}) \in H_{\chi}(p)$, let $\underline{R}^{(n)}:=$ the admissible concatenation of $w_{n}$ to itself. Then $\underline{R}^{(n)} \rightarrow \underline{R}$. As demonstrated in the proof of Proposition 4.11, there exists $\underline{u}^{(n)}, \underline{u} \in \stackrel{\Sigma}{\star}^{\#}$ such that $\underline{u}^{(n)} \rightarrow \underline{u}$ and $\pi\left(\underline{u}^{(n)}\right)=$ $\widehat{\pi}\left(\underline{R}^{(n)}\right), \pi(\underline{u})=\widehat{\pi}(\underline{R})$. It follows that $V^{u}\left(\underline{u}^{(n)}\right) \rightarrow V^{u}(\underline{u}), V^{s}\left(\underline{u}^{(n)}\right) \rightarrow V^{s}(\underline{u})$ as admissible manifolds in $u_{0}$ (i.e. the representing functions converge in $C^{1}$-norm).

Since $\pi(\underline{u})=\widehat{\pi}(\underline{R}) \in H_{\chi}(p)$, there exists $N=N_{\underline{R}}$ such that $f^{N}\left[V^{u}\left(\sigma^{-N} \underline{u}\right)\right] \pitchfork$ $W^{s}(o(p)) \neq \varnothing, f^{-N}\left[V^{s}\left(\sigma^{N} \underline{u}\right)\right] \pitchfork W^{s}(o(p)) \neq \varnothing$. So, there exists $n_{\underline{R}}$ such that for all $n \geq n_{\underline{R}}, f^{N}\left[V^{u}\left(\sigma^{-N} \underline{u}^{(n)}\right)\right] \pitchfork W^{s}(o(p)) \neq \varnothing, f^{-N}\left[V^{s}\left(\sigma^{N} \underline{u}^{(n)}\right)\right] \pitchfork W^{s}(o(p)) \neq \varnothing$. 
Let $\quad P_{\underline{R}}:=\left\{x_{\underline{R}}^{(n)}\right\}_{|n|>n_{\underline{R}}}$. Then for all $n \geq n_{\underline{R}}, \quad x_{\underline{R}}^{(n)} \in H_{\chi}(p)$, and therefore $P_{\underline{R}} \subseteq H_{\chi}(p)$.

Consider the countable collection of all periodic points generated in this manner $\left\{p_{i}\right\}_{i \geq 0}=\bigcup\left\{P_{\underline{R}}: \underline{R} \in \widehat{\Sigma}^{\# \#} \cap \widehat{\pi}^{-1}[\{x\}], x \in H_{\chi}(p)\right\}\left(\subseteq \mathrm{RWT}_{\chi}^{\star}\right.$, Theorem 4.5). Then by the transitivity of the homoclinic relation [New89, Proposition 2.1], for all $i, j \geq 0$, $p_{i} \in H_{\chi}\left(p_{j}\right)$. Assume without loss of generality that there exists $N_{l} \uparrow \infty$ such that for all $l \in \mathbb{N}$, for all $i \leq N_{l}, o\left(p_{i}\right) \subseteq\left\{p_{j}\right\}_{j \leq N_{l}}$. Fix $N \in\left\{N_{l}\right\}_{l \geq 0}$.

Step 1. For all $0 \leq i, j \leq N$ there exists $t_{i j} \in\left(W^{u}\left(p_{i}\right) \pitchfork W^{s}\left(p_{j}\right)\right) \cap H_{\chi}(p)$, and $t_{i j}$ has a uniformly hyperbolic orbit, and its coding involves finitely many letters (i.e. for all $t_{i j}$ there exists $\left\{R_{l}^{(i j)}\right\}_{l=1}^{m}, \underline{R}^{(i j)} \in \widehat{\widehat{\Sigma}} \cap\left(\left\{R_{l}^{(i j)}\right\}_{l=1}^{m}\right)^{\mathbb{Z}}$ such that $\left.\widehat{\pi}\left(\underline{R}^{(i j)}\right)=t_{i j}\right)$.

Proof of step 1. (a) The orbit of $t_{i j}$ is uniformly hyperbolic. Since $p_{i} \in H_{\chi}\left(p_{j}\right)$, there exists $t_{i j} \in W^{u}\left(p_{i}\right) \pitchfork W^{s}\left(p_{j}\right)$. Showing that $t_{i j}$ has a uniformly hyperbolic orbit would yield that $t_{i j} \in \mathrm{RWT}_{\chi}^{\star}$, and so, since $p_{i}, p_{j} \in H_{\chi}(p)$, also $t_{i j} \in H_{\chi}(p)$. By Lemma 5.4, $p_{i}, p_{j} \in \chi$-hyp; hence, by [BO18, Lemma 4.5], $t_{i j} \in \chi$-hyp. By the inclination lemma [BS02, Theorem 5.7.2], the angle between $W^{s}\left(t_{i j}\right)$ and $W^{u}\left(t_{i j}\right)$ is bounded away from zero along the orbit of $t_{i j}$. Therefore, by [BO18, Lemma 4.5] and by Lemma 5.6, $\left\{\left\|C_{\chi}^{-1}\left(f^{k}\left(t_{i j}\right)\right)\right\|\right\}_{k \in \mathbb{Z}}$ is bounded along the orbit of $t_{i j}$. Thus, $t_{i j} \in \mathrm{RWT}_{\chi}^{\star}$ and has a uniformly hyperbolic orbit.

(b) Coding of a uniformly hyperbolic orbit with finitely many letters. Assume $x \in$ $\mathrm{RWT}_{\chi}^{\star}$ has a uniformly hyperbolic orbit (i.e. $\left\{\left\|C_{\chi}^{-1}\left(f^{n}(x)\right)\right\|\right\}_{n \in \mathbb{Z}}$ is bounded). By [Sar13, Proposition 4.5], there exists a chain $\underline{u}=\left\{\psi_{x_{i}}^{p_{i}^{s}, p_{i}^{u}}\right\}_{i \in \mathbb{Z}} \in \Sigma^{\star} \#$ such that $\pi(\underline{u})=x$, $Q_{\epsilon}\left(x_{i}\right) / Q_{\epsilon}\left(f^{i}(x)\right)=e^{ \pm \epsilon / 3}$ for all $i \in \mathbb{Z}$, and $p_{i}^{u}, p_{i}^{s}$ are given by a formula such that

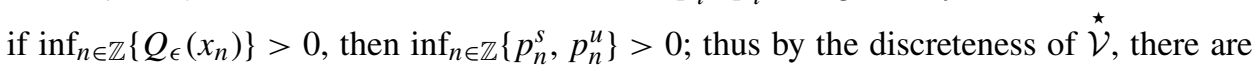
finitely many possible letters in that coding. For all $i \in \mathbb{Z}, R\left(f^{i}(x)\right) \subseteq \stackrel{\star}{Z}\left(u_{i}\right)$, and so, by Theorem 4.8, $\underline{R}(x)$ contains finitely many letters. In addition, for all $\underline{R} \in \widehat{\widehat{\Sigma}}^{\star} \cap \widehat{\pi}^{-1}[\{x\}]$, for all $i \in \mathbb{Z}, R_{i}$ and $R\left(f^{i}(x)\right)$ are $\epsilon$-affiliated; thus $\underline{R}$ contains only finitely many letters as well (see remark after Definition 4.9).

Step 2. Let $\left\{t_{i j}\right\}_{i, j \leq N}$ be defined as in step 1 , and choose some $\zeta_{i j} \in \widehat{\pi}^{-1}\left[\left\{t_{i j}\right\}\right]$ which involves only finitely many letters. As defined before step 0 , for all $p \in\left\{p_{i}\right\}_{i \geq 0}, p=$ $\widehat{\pi}(\underline{w}(p))$ where $\underline{w}(p) \in \widehat{\widehat{\Sigma}}^{\star} \#$ and there exists $m_{\underline{w}(p)} \geq 0$ such that $\sigma^{m_{\underline{w}}(p)} \underline{w}(p)=\underline{w}(p)$. Let

$$
\tilde{L}:=\left\{\underline{w}\left(p_{i}\right): 0 \leq i \leq N\right\} \cup\left\{\underline{\zeta}_{i j}\right\}_{i, j \leq N} .
$$

$\tilde{L}$ is finite and involves only finitely many letters.

Step 3. Define

$$
L:=\bigcup_{\underline{u} \in \tilde{L}}\left\{\sigma^{j} \underline{u}\right\}_{j \in \mathbb{Z}} .
$$

By the Hölder continuity of $\widehat{\pi}$ (Theorem 4.10), for all $y \in \widehat{\pi}[L]$, there exist $j^{+}(y)$, $j^{-}(y) \leq N \quad$ such that $\lim _{n \rightarrow \infty} d\left(f^{-n}(y), f^{-n}\left(p_{j^{-}(y)}\right)\right)=0, \lim _{n \rightarrow \infty} d\left(f^{n}(y)\right.$, 
$\left.f^{n}\left(p_{j^{+}(y)}\right)\right)=0$. Therefore, $\widehat{\pi}[L]$ is compact, $f$-invariant, and $\chi^{\prime}$-uniformly hyperbolic for some $\chi^{\prime}>\chi$ (by the proof of step 1).

Step 4. We now follow the argument in [BCS, Lemma 3.12], By the shadowing lemma, there are $\epsilon^{\prime}>0, \delta>0$ such that:

(1) every $\epsilon^{\prime}$-pseudo-orbit in $L^{\mathbb{Z}}$ is $\delta$-shadowed by at least one real orbit [KH95, Theorem 18.1.2];

(2) every $\epsilon^{\prime}$-pseudo-orbit in $L^{\mathbb{Z}}$ is $2 \delta$-shadowed by at most one orbit by expansivity; see [KH95, Theorem 18.1.3] (in particular, every orbit as in the first item is unique).

Since $\tilde{L}$ is finite, there is some $m \geq 0$ large enough so that $d\left(f^{m}(y), f^{m}\left(p_{j^{+}(y)}\right)\right)<$ $\epsilon^{\prime} / 2, d\left(f^{-m}(y), f^{-m}\left(p_{j^{-}(y)}\right)\right)<\epsilon^{\prime} / 2$, for all $y \in \widehat{\pi}[\tilde{L}]$. Let $L_{m}:=\bigcup_{j=-m}^{m}\left\{f^{j}(y): y \in\right.$ $\widehat{\pi}[\tilde{L}]\}$, which is also finite. Let

$$
K:=\left\{x \in M: \text { the orbit of } x \text { is } \delta \text {-shadowed by an } \epsilon^{\prime} \text {-pseudo-orbit in } L_{m}^{\mathbb{Z}}\right\} .
$$

This set contains $\widehat{\pi}[\tilde{L}]$, and since $L_{m}$ is finite, it is also closed. It is also invariant and uniformly $\chi^{\prime}$-hyperbolic for some $\chi^{\prime}>\chi$ (whence $\subseteq \mathrm{RWT}_{\chi}^{\star}$ ).

We construct a point in $K$ with a dense forward orbit in $K$ in the following way. Take a list of all finite $\epsilon^{\prime}$-pseudo-orbits $\left\{\omega_{i}\right\}_{i \geq 0}$ with letters in $L_{m}$. Each $y \in L_{m}$ connects by an admissible word of length at most $m$ to some periodic point $p_{j^{+}(y)} \in L_{m}$, and has some periodic point $p_{j^{-}(y)}$ connecting to it by an admissible word of length at most $m$. For each two periodic points $p_{i}, p_{j} \in L_{m}, p_{i}$ connects to $p_{j}$ by an admissible word of length at most $2 m$ through $t_{i j}$. Therefore, every pair of admissible finite words $\omega, \omega^{\prime}$ of letters in $L_{m}$ can be concatenated by some admissible finite word of letters in $L_{m}$. Concatenate this way all words in $\left\{\omega_{i}\right\}_{i \geq 0}$, and take any admissible continuation to the past. This yields an $\epsilon^{\prime}$-pseudo-orbit, and the unique orbit in $K$ it $\delta$-shadows must be dense in $K$ by expansivity; denote this orbit by $o(x)$. This can be seen by [Bow08, Lemma 3.13]: the mapping $\tau$ which maps each $\epsilon^{\prime}$-pseudo-orbit to its uniquely $\delta$-shadowed orbit is a continuous map (the topology on the space of pseudo-orbits is the metric topology generated by cylinders); and in addition $\tau \circ \sigma=f \circ \tau$, where $\sigma$ denotes the left-shift on $\epsilon^{\prime}$-pseudo-orbits. Thus, shadowing longer intervals of the orbit of $x$ forces to be closer to it.

Step 5. The orbit of $x$ lies in $K$, which is an invariant $\chi^{\prime}$-uniformly hyperbolic set for some $\chi^{\prime}>\chi$ (see [KH95, Proof of Proposition 6.4.6]), whence $\left\|C_{\chi}^{-1}(\cdot)\right\|$ is uniformly bounded on $K$; and by the same argument as part (b) of step 1, $x$ has a pre-image in $\widehat{\widehat{\Sigma}}^{\#}$ which contains only finitely many letters. Choose one pre-image like that, and denote it by $\underline{v}$. There exists at least one symbol $v^{\prime}$ such that the forward orbit of $\underline{v}$ visits its cylinder $\left[v^{\prime}\right]$ infinitely often. Let $\underline{v}^{+}:=\sigma^{\min \left\{i \geq 0: \sigma^{i} \underline{v} \in\left[v^{\prime}\right]\right\}} \underline{v}$, and let $\underline{v}^{-}$be some periodic chain in $\left[v^{\prime}\right]$. Define

$$
v_{i}^{\prime}:=\left\{\begin{array}{ll}
v_{i}^{+} & \text {if } i \geq 0, \\
v_{i}^{-} & \text {if } i \leq 0,
\end{array} \quad \text { and write } x^{\prime}:=\widehat{\pi}\left(\underline{v}^{\prime}\right)\right.
$$

Then the forward orbit of $x^{\prime}$ is dense in $K$, and $\underline{v}^{\prime} \in \widetilde{\Sigma}_{N}:=\left\{\underline{u} \in \widehat{\widehat{\Sigma}}: \underline{u} \in\left\langle v^{\prime}\right\rangle^{\mathbb{Z}}\right\}$ (recall Definition 5.3). $\widetilde{\Sigma}_{N}$ is a maximal irreducible component of $\widehat{\Sigma}$ containing a compact set which contains the orbit of $\underline{v}^{\prime}$. For each $y \in \widehat{\pi}[\tilde{L}] \subseteq K$, the orbit of $x^{\prime}$ has a subsequence converging to it. Since $\underline{v}^{\prime}$ is made of finitely many letters, the orbit of $\underline{v}^{\prime}$ belongs to a 
compact subset of $\widetilde{\Sigma}_{N}$. Therefore, the subsequence $\left\{f^{n_{k}}\left(x^{\prime}\right)\right\}_{k \geq 0}$ which converges to $y$, has a subsubsequence of its own such that $\left\{\sigma^{n{ }_{j}} \underline{v}^{\prime}\right\}_{j \geq 0}$ converges as well. By the continuity of $\widehat{\pi}$, that limit must code $y$. Therefore $\widehat{\pi}\left[\widetilde{\Sigma}_{N}\right] \supseteq \widehat{\pi}[\tilde{L}]$ and, moreover, each term in $\widehat{\pi}[\tilde{L}]$ has a pre-image in $\widetilde{\Sigma}_{N}$ made of finitely many letters.

Step 6. For each $N \in\left\{N_{l}\right\}_{l \geq 0}, p_{0} \in \widehat{\pi}\left[\widetilde{\Sigma}_{N}\right]$. Since $\left.\widehat{\pi}\right|_{\widehat{\Sigma}^{\#}}$ is finite-to-one, there could be only finitely many maximal irreducible components in $\stackrel{\Sigma}{\Sigma}$, which can code $p_{0}$. Therefore, there is some subsequence of the maximal irreducible components from step $5, \widetilde{\Sigma}_{N_{l_{j}}}$, $l_{j} \uparrow \infty$, which is constant. Denote this component by $\widetilde{\Sigma}$. For each fixed $N \in\left\{N_{l}\right\}_{l \geq 0}$, we constructed a set $\tilde{L}$, and all such sets must be covered by $\widehat{\pi}[\widetilde{\Sigma}]$.

Step 7. Given a point $z \in H_{\chi}(p) \cap \widehat{\pi}\left[\widehat{\Sigma}^{\# \#}\right]$, consider its coding $\underline{S}$, as in equation (8). This coding has a sequence of periodic chains $\left\{\underline{S}^{(n)}\right\}_{n \geq n_{S}} \subseteq\left[S_{0}\right]$, which converge to it. For all $n \geq n_{\underline{S}}, z_{\underline{S}}^{(n)}:=\widehat{\pi}\left(\underline{S}^{(n)}\right)$ can be coded by a chain in $\widetilde{\Sigma}$ with finitely many different letters. These chains (which contain finitely many letters, and as such lie in $\widehat{\widehat{\Sigma}}^{\#}$ ) all belong to a cylinder from $\left\{[b] \in \stackrel{\star}{\mathcal{R}}: b\right.$ is $\epsilon$-affiliated to $\left.S_{0}\right\}$, which is a finite collection (see the remark after Definition 4.9). Thus they have a converging subsequence with a limit in $\widetilde{\Sigma}$ (cylinders are compact since $\widehat{\star}$ is locally compact [Sar13, $\S 12.2]$, and $\widetilde{\Sigma}$ is a closed subset). By the continuity of $\widehat{\pi}$, that limit must code $z$, and so $\widehat{\pi}[\widetilde{\Sigma}] \supseteq H_{\chi}(p) \cap \widehat{\pi}\left[\widehat{\Sigma}^{\# \#}\right]=$ $H_{\chi}(p)$ modulo all conservative measures which are carried by $H_{\chi}(p)$.

We compare our proof to the one in [BCS]. In [BCS, Lemma 3.13], the authors give a procedure for lifting invariant probability measures which are carried by $H_{\chi}(p)$ to an irreducible coding. Their technique involves restriction to points which are generic with respect to Birkhoff's ergodic theorem, and it therefore does not apply to infinite measures. The lifting is done by the formula $\widehat{\mu}:=\int\left(1 /\left|N_{x}\right|\right) \sum_{\underline{R} \in N_{x}} \delta_{\underline{R}} d \mu(x)$, where $N_{x}:=\widehat{\pi}^{-1}[\{x\}] \cap \widetilde{\Sigma}^{\#} \quad$ and $\quad \widetilde{\Sigma}^{\#}:=\left\{\underline{u} \in \widetilde{\Sigma}\right.$ : there exists $v, w$ such that $\#\left\{i>0: u_{i}=\right.$ $v\}=\infty$, and $\left.\#\left\{i<0: u_{i}=w\right\}=\infty\right\}$. When $\mu$ is carried by $\widehat{\pi}\left[\widetilde{\Sigma}^{\#}\right]$, this lifting is well defined since $\widehat{\pi}$ is finite-to-one on $\widehat{\Sigma}^{\star}$. So, we are required to find a different way which does not depend on generic points to show that all conservative measures which are carried by $H_{\chi}(p)$ are also carried by $\widehat{\pi}\left[\widetilde{\Sigma}^{\#}\right]$.

The main difficulty in doing so is as follows. Let there be a point $x \in H_{\chi}(p) \cap \widehat{\pi}\left[\widehat{\widehat{\Sigma}}^{\# \#}\right]$ which can be coded by a chain $\underline{u} \in \widetilde{\Sigma}$, and assume that $\underline{u}$ is the limit of a sequence $\underline{u}^{(n)} \in$

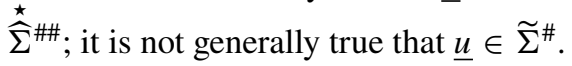

The chains which are shown to code $H_{\chi}(p) \cap \widehat{\pi}\left[\widehat{\Sigma}^{\# \#}\right]$ in Proposition 5.8 were constructed in such a way as to guarantee that we can overcome that difficulty.

In [BCS, Lemma 3.13], the homoclinic class which is being lifted to an irreducible component (modulo all invariant ergodic probability measures carried by it) is $H O(p):=$

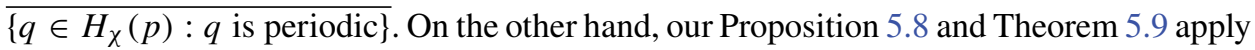
to ergodic homoclinic classes in the sense of [RHRHTU11], which are smaller. The reason for this is that ergodic homoclinic classes are relevant to specific objects of our interest (e.g. SRB measures on ergodic homoclinic classes; see [RHRHTU11]), and we find them easier to study, and their notion more suitable for our needs. 
THEOREM 5.9. Let $p$ be a $\chi$-hyperbolic periodic point. Let $\widetilde{\Sigma}$ be the irreducible TMS from Proposition 5.8 which covers $H_{\chi}(p)$ modulo all conservative measures. Then $\widehat{\pi}\left[\widetilde{\Sigma}^{\#}\right]=$ $H_{\chi}(p)$ modulo all conservative measures which are carried by $H_{\chi}(p)$.

Proof. $p$ is clearly in $H_{\chi}(p) \cap \widehat{\pi}\left[\widehat{\Sigma}^{\# \#}\right]$, and so it is has a coding in $\widetilde{\Sigma}$. For all $\underline{v} \in \widetilde{\Sigma}^{\#}$, $\widehat{\pi}(\underline{v}) \in H_{\chi}(p)$ by the irreducibility of $\widetilde{\Sigma}$ (and since $\widehat{\pi}\left[\widehat{\Sigma}^{\#}\right]=\operatorname{RWT}_{\chi}^{\star}$ ), thus the inclusion $\subseteq$ is clear. It remains to show the inclusion $\supseteq$. Given each $x \in H_{\chi}(p) \cap \widehat{\pi}\left[\widehat{\widehat{\Sigma}}^{\# \#}\right]\left(=H_{\chi}(p)\right.$ modulo all conservative measures on $\left.H_{\chi}(p)\right), x$ has a coding $\underline{v} \in \widehat{\widehat{\Sigma}}^{\# \#}$ as in equation (8). In step 0 of Proposition 5.8 we described a sequence of periodic chains which converge to $\underline{v}$. Call these chains $\left\{\underline{v}^{(i)}\right\}_{i \geq 0}$. By step 5 of Proposition 5.8, $\left\{\widehat{\pi}\left(\underline{v}^{(i)}\right)\right\}_{i \geq 0}$ all have codings made of finitely many letters in $\widetilde{\Sigma}$, denoted by $\left\{\underline{u}^{(i)}\right\}_{i \geq 0}$, and by step 7 of Proposition 5.8 we can assume without loss of generality that $\left\{\underline{u}^{(i)}\right\}_{i \geq 0}$ converge to some limit $\underline{u} \in \widetilde{\Sigma}$, with $\widehat{\pi}(\underline{u})=x$. Let $\left(v_{j_{l}}\right)_{l \in \mathbb{N}}$ be a constant subsequence of $\left(v_{i}\right)_{i \geq 0}$ (it exists since $\underline{v} \in \widehat{\Sigma}^{\star}$ ); denote by $w$ the symbol which satisfies $v_{j_{l}}=w$ for all $l \geq 0$. For all $l \geq 0$ there exists $i_{l}$ such that for all $i>i_{l}, v_{j_{l}}^{(i)}=v_{j_{l}}=w$. If $i$ is big enough that $d\left(\underline{u}^{(i)}, \underline{u}\right) \leq e^{-j_{l}}$, then $u_{j_{l}}^{(i)}=$ $u_{j_{l}} \cdot \underline{v}^{(i)}, \underline{u}^{(i)}$ both code the same point and lie in $\widehat{\Sigma}^{\star}$, therefore $u_{j_{l}}^{(i)}$ is $\epsilon$-affiliated to $v_{j_{l}}^{(i)}$. Therefore, $w$ is $\epsilon$-affiliated to $u_{j_{l}}$. Since $\#\left\{v^{\prime}: v^{\prime}\right.$ is $\epsilon$-affiliated to $\left.w\right\}<\infty$, we get by the pigeonhole principle that some symbol must repeat in $\underline{u}$ for infinitely many positive indices. Similarly, it follows that some symbol must repeat in $\underline{u}$ for infinitely many negative indices. Therefore, $\underline{u} \in \widetilde{\Sigma}^{\#}$ and $\widehat{\pi}(\underline{u})=x$.

Acknowledgements. This work constitutes part of a doctoral thesis, conducted at the Weizmann Institute of Science under the guidance of O. Sarig. While working on this project, I benefited from discussions with Jérôme Buzzi and Sylvain Crovisier. I would also like to thank Yuri Lima for reading the paper with care and making useful remarks, and the Weizmann Institute of Science for excellent working conditions. In addition, I would like to thank the referee and Yuri Lima for finding a mistake in an earlier version of this paper. This research was partially supported by ISF grant 1149/18 and BSF grant 2016105.

\section{REFERENCES}

[Aar97] J. Aaronson. An Introduction to Infinite Ergodic Theory (Mathematical Surveys and Monographs, 50). American Mathematical Society, Providence, RI, 1997.

[AW67] R. L. Adler and B. Weiss. Entropy, a complete metric invariant for automorphisms of the torus. Proc. Natl. Acad. Sci. USA 57 (1967), 1573-1576.

[BCS] J. Buzzi, S. Crovisier and O. Sarig. Measures of maximal entropy for surface diffeomorphisms. Preprint, 2018, arXiv:1811.02240.

[BO18] S. Ben Ovadia. Symbolic dynamics for non-uniformly hyperbolic diffeomorphisms of compact smooth manifolds. J. Mod. Dyn. 13 (2018), 43-113.

[Bow08] R. Bowen. Equilibrium States and the Ergodic Theory of Anosov Diffeomorphisms (Lecture Notes in Mathematics, 470), revised edn. With a preface by David Ruelle. Ed. J.-R. Chazottes. Springer, Berlin, 2008.

[BR75] R. Bowen and D. Ruelle. The ergodic theory of Axiom A flows. Invent. Math. 29(3) (1975), 181-202. 
[BS02]

[Kat80]

[KH95]

[KM95]

[Lim]

[Lim19]

[Lim20]

[LM18]

[LS19]

[New72]

[New89]

[Pes76]

[PP90]

[RHRHTU11]

[Rue76]

[Rue04]

[Sar13]

[Sin68a]

[Sin68b]

[Sin72]
M. Brin and G. Stuck. Introduction to Dynamical Systems. Cambridge University Press, Cambridge, 2002.

A. Katok. Lyapunov exponents, entropy and periodic orbits for diffeomorphisms. Publ. Math. Inst. Hautes Études Sci. 51 (1980), 137-173.

A. Katok and B. Hasselblatt. Introduction to the Modern Theory of Dynamical Systems (Encyclopedia of Mathematics and its Applications, 54). Cambridge University Press, Cambridge, 1995 . With a supplementary chapter by Katok and Leonardo Mendoza.

A. Katok and L. Mendoza. Dynamical systems with non-uniformly hyperbolic behavior. Supplement to Introduction to the Modern Theory of Dynamical Systems (Encyclopedia of Mathematics and its Applications, 54). Cambridge University Press, Cambridge, 1995.

Y. Lima. Private communication.

Y. Lima. Symbolic dynamics for nonuniformly hyperbolic systems. Preprint, 2019, arXiv:1910.11371.

Y. Lima. Symbolic dynamics for one dimensional maps with nonuniform expansion. Ann. Inst. H. Poincaré Anal. Non Linéaire, 37(3) (2020), 727-755.

Y. Lima and C. Matheus. Symbolic dynamics for non-uniformly hyperbolic surface maps with discontinuities. Ann. Sci. Éc. Norm. Supér. (4) 51(1) (2018), 1-38.

Y. Lima and O. M. Sarig. Symbolic dynamics for three-dimensional flows with positive topological entropy. J. Eur. Math. Soc. (JEMS) 21(1) (2019), 199-256.

S. E. Newhouse. Hyperbolic limit sets. Trans. Amer. Math. Soc. 167 (1972), 125-150.

S. E. Newhouse. Continuity properties of entropy. Ann. of Math. (2) 129(2) (1989), 215-235.

J. B. Pesin. Families of invariant manifolds that correspond to nonzero characteristic exponents. Izv. Akad. Nauk SSSR Ser. Mat. 40(6) (1976), 1332-1379, 1440.

W. Parry and M. Pollicott. Zeta functions and the periodic orbit structure of hyperbolic dynamics. Astérisque 268 (1990), 187-188.

F. Rodriguez Hertz, M. A. Rodriguez Hertz, A. Tahzibi and R. Ures. Uniqueness of SRB measures for transitive diffeomorphisms on surfaces. Comm. Math. Phys. 306(1) (2011), 35-49. D. Ruelle. A measure associated with axiom-A attractors. Amer. J. Math. 98 (1976), 619-654.

D. Ruelle. Thermodynamic formalism. The Mathematical Structures of Equilibrium Statistical Mechanics (Cambridge Mathematical Library), 2nd edn. Cambridge University Press, Cambridge, 2004.

O. M. Sarig. Symbolic dynamics for surface diffeomorphisms with positive entropy. J. Amer. Math. Soc. 26(2) (2013), 341-426.

J. G. Sinaŭ. Construction of Markov partitionings. Funktsional. Anal. i Priložhen. 2(3) (1968), 70-80 (Loose errata).

J. G. Sinaĭ. Markov partitions and U-diffeomorphisms. Funktsional. Anal. i Priložhen. 2(1) (1968), 64-89.

J. G. Sinaŭ. Gibbs measures in ergodic theory. Uspekhi Mat. Nauk 27(4(166)) (1972), 21-64. 